Historic, archived document

Do not assume content reflects current scientific knowledge, policies, or practices. 


$$
-
$$




\section{MAULE'S SPECIAL}

WHOLESALE PRICE LIST

Vegetable and Flower Seeds Bulbs and Roots for

MARKET GROWERS and FLORISTS

$$
1933 \text { TEGETABLA }
$$

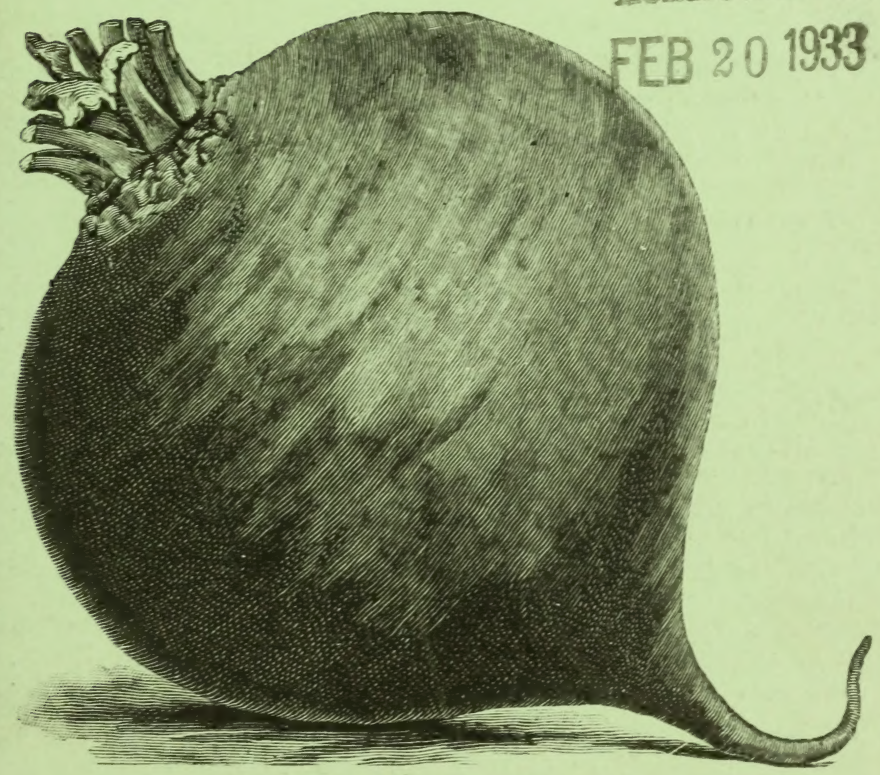

Maule's Blood Turnip Beet

WM. HENRY MAULE

"The Old Reliable Seed House" 1220 Spring Garden Street 


\section{$\stackrel{1877}{\longrightarrow}$ MAULE'S SEEDS $\underline{\overline{1933}}$}

"THERE ARE NONE BETTER"

We take great pleasure in sending you this Special Wholesale Price List for Market Gardeners and Florists. You will find listed on the following pages very attractive low prices which we feel you are entitled to. We are confident that these prices will meet with your approval, and will have as great a response as last year, which was our most successful Market Gardener season.

\section{THIS BOOK IS A READY REFERENCE PRICE LIST}

not a catalog. For full description of the seeds, bulbs, and roots, see Maule's Seed Book for 1933, a copy of which has been sent you. If you have not received your copy, kindly advise us, and another copy will be mailed you free.

\section{Plant Maule's Seeds for Success}

Each lot of seed is thoroughly tested for active, abundant and vigorous life so that only the most virile product is shipped. The exacting nature of these tests has won the confidence of thousands and thousands of gardeners who not only buy Maule's Seeds year after year but gladly recommend them to their friends. Many new customers are added to our list each year through recommendations of those who know just how good Maule's Seeds really are because they have planted them.

\section{Send in Your Order Early}

We know our old Market Gardener and Florist customers will favor us with their orders again. We hope for a trial order, no matter how small or large, from every new reader of this Price List. Glance through these pages and prove to your own satisfaction that our prices are right. Then send us your order and let us prove to you that while there may be other Seeds, Bulbs, and Roots as good as Maule's “There Are None Better."

\section{Terms: Cash With Order}

Our terms are cash with order, but if you want your order sent by parcel post or express C. O. D., or freight, sight draft bill of lading attached, 25 per cent. of the amount should accompany the order. All C. O. D. charges should be assumed by customer.

\section{Maule Pays the Postage or Express Charges On All Seeds}

Except Where Noted Otherwise

We pay the postage on all Flower Seeds in any quantity. All Vegetable Seeds in any quantity listed Postpaid or Express Prepaid.

Beans, Sweet Corn and Peas in any quantity up to $10 \mathrm{lbs}$. listed Postpaid or Express Prepaid.

On the following, listed "Not Prepaid," the purchaser pays the transportation charges.

Beans, Sweet Corn, Peas, Asparagus Roots, Horse Radish Sets, Mushroom Spawn, Onion Sets, Potatoes, Rhubarb Roots, and Flowering Bulbs.

\section{Our Guarantee to You}

\section{We Guarantee:}

All goods shipped by us to reach you in good condition.

To refill your order or refund, any time within the year, (whichever you prefer) the full amount paid us for Seeds, Bulbs, or Roots, if you are not satisfied with results.

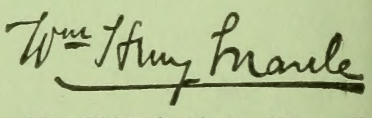

"The Old Reliable Seed House" 


\section{Maule's Superior Vegetable Seeds}

\section{Seeds Delivered by Parcel Post or Express, Free of all Charges Except Where Noted}

All seeds offered in any quantity listed Postpaid or Express Prepaid in this Special Price List will be delivered to your home, post office, or express office free of all charges.

100 lb. lots of Beans, Sweet Corn, Peas; Mushroom Spawn, Onion. Sets, Potatoes, Bulbs and Roots, listed not prepaid. Customers pay_transportation charges on these goods.

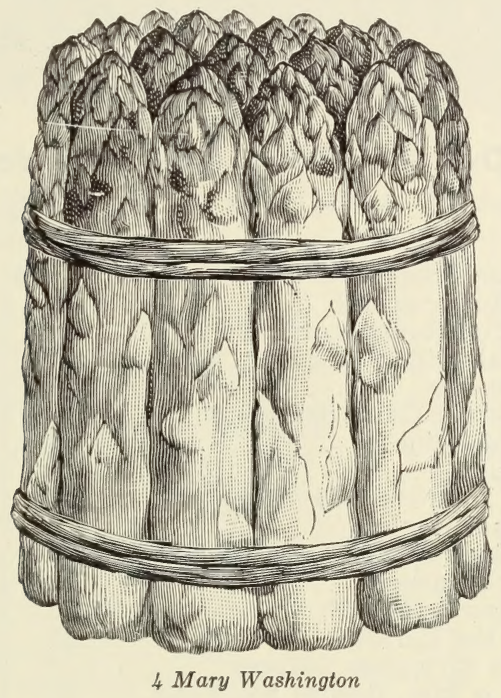

ASPARAGUS SEED

4 Mary Washington $\frac{5}{1 / 4} \mathrm{lb} .1 \mathrm{lb} .5 \mathrm{lbs} .10 \mathrm{lbs} .100 \mathrm{lbs}$ $\begin{array}{llllll}\text { Rust Resistant... ... } \$ 0.30 & \$ 0.80 & \$ 3.75 & \$ 6.50 & \$ 62.50\end{array}$

\section{ASPARAGUS ROOTS}

NOTE-You may order 2,000 roots or over at the 5,000 rate

\begin{tabular}{|c|c|}
\hline & Not Prepaid \\
\hline Mary Wa & $\begin{array}{ccc}250 & 1000 & 5000 \\
\text { Roots } & \text { Roots } & \text { Roots }\end{array}$ \\
\hline One-yea & $\$ 8.00 \$ 37.50$ \\
\hline ant, $\mathrm{Tr}$ & 40.00 \\
\hline
\end{tabular}
Asparagus Roots are out of season from June to October.

\section{ARTICHOKE}

2 Green Globe............... \$0.25 $\$ 0.40 \quad \$ 1.35 \quad \$ 4.50$

\section{BROCCOLI}

Postpaid or Express Prepaid

103 Early Angiers. $1 / 2$ oz. 1 oz. $\quad 1 / 4 \mathrm{lb} .1 \mathrm{lb}$.

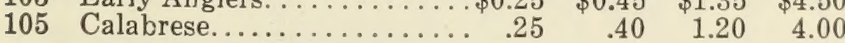

\section{BRUSSELS SPROUTS}




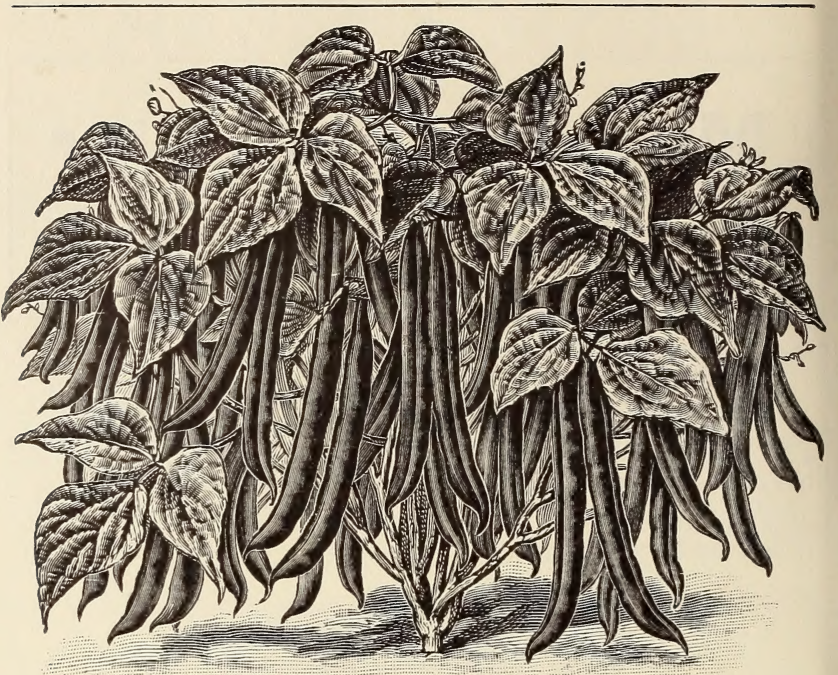

10 Mammoth or Giant Stringless Bean

BEANS-Dwarf or Bush, Green Podded

\begin{tabular}{|c|c|c|c|c|}
\hline & & $\begin{array}{r}\text { Postr } \\
\text { Express }\end{array}$ & $\begin{array}{l}\text { paid or } \\
\text { Prepaid }\end{array}$ & $\underset{\text { Not }}{\text { Prepaid }}$ \\
\hline & & 5 lbs. & $10 \mathrm{lbs}$. & $100 \mathrm{lbs}$. \\
\hline & Tennessee Green-Pod & $\$ 1.05$ & $\$ 1.95$ & $\$ 11.00$ \\
\hline & Dwarf Hort & .1 .10 & 2. & 12.00 \\
\hline & ee or 1,000 to & .95 & & 9.00 \\
\hline & Cot. & 1.10 & & 14. \\
\hline & $\mathrm{Fu}$ & 1.10 & & 12.0 \\
\hline & & 1. & & \\
\hline & & & & \\
\hline & & & & \\
\hline & & & & \\
\hline & & & & \\
\hline & & & & \\
\hline & & & & \\
\hline & $\mathrm{Lc}$ & & & \\
\hline & & & & \\
\hline & & & & \\
\hline & Ro & .90 & & \\
\hline & & 1. & & \\
\hline & & 1.20 & & \\
\hline & Tendergreen Stringless. & 1.10 & 2.00 & \\
\hline
\end{tabular}

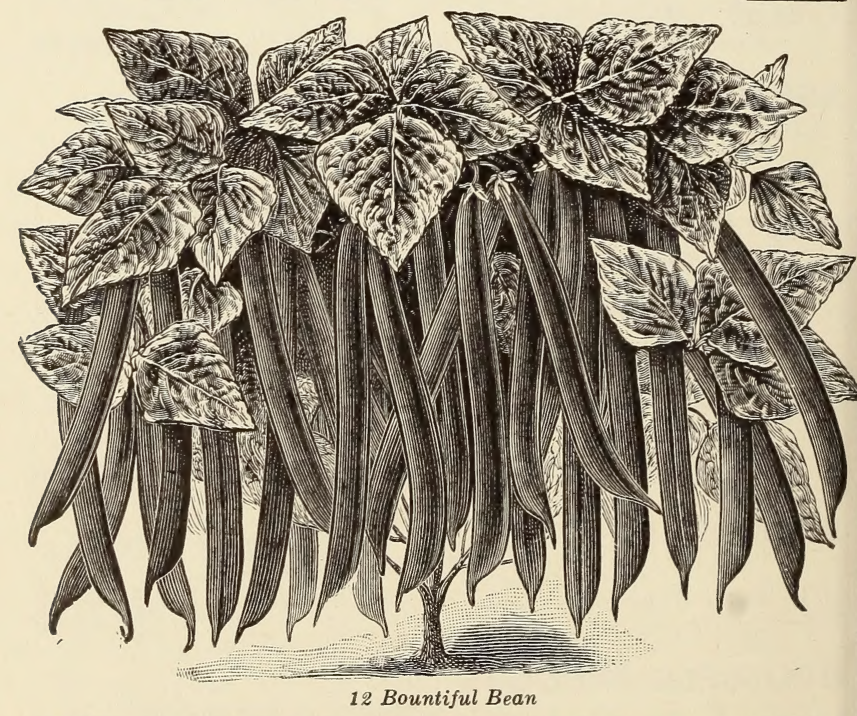

REMEMBER ${ }_{\text {BAGS, CRATES, OR PACKING }}^{\text {WE MAKE NO CHARE FOR }}$

NOTE-All Beans you may order 2 lbs. or over at $5 \mathrm{lb}$. rate; 6 lbs. or over at $10 \mathrm{lb}$. rate, postpaid or express prepaid; $15 \mathrm{lbs}$. or over at $100 \mathrm{lb}$. rate, not prepaid. 


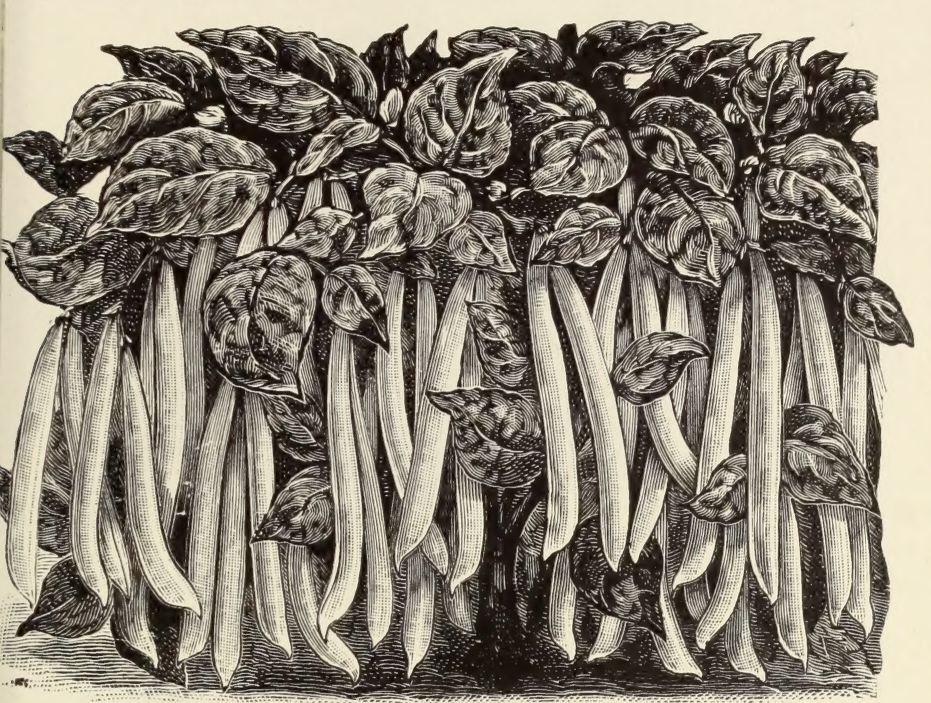

27 Maule's Improved Butter Wax Bean

\begin{tabular}{|c|c|c|c|c|}
\hline \\
\hline & \\
\hline & & & & 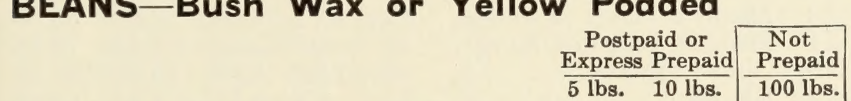 \\
\hline \\
\hline \multirow{2}{*}{\multicolumn{5}{|c|}{ 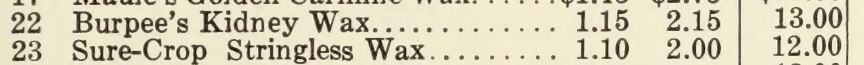 }} \\
\hline & & & & \\
\hline \multicolumn{5}{|c|}{ 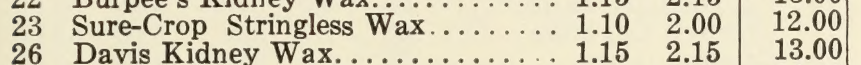 } \\
\hline \multicolumn{5}{|c|}{7 Maule's Improved Butter Wax...... 1.20} \\
\hline \multicolumn{5}{|c|}{ 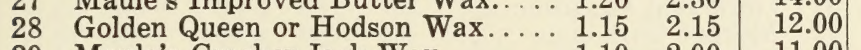 } \\
\hline \multirow{2}{*}{\multicolumn{5}{|c|}{ 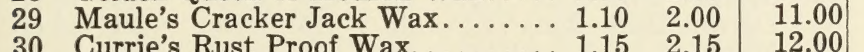 }} \\
\hline & & & & \\
\hline \multicolumn{5}{|c|}{ 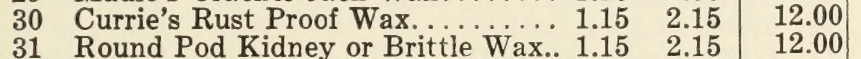 } \\
\hline \multicolumn{5}{|c|}{ 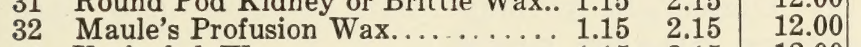 } \\
\hline \multirow{2}{*}{\multicolumn{5}{|c|}{ 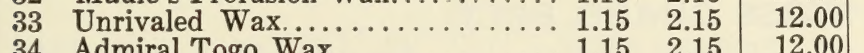 }} \\
\hline & & & & \\
\hline & & \multirow{2}{*}{$\begin{array}{l}1.15 \\
1.15 \\
1.10\end{array}$} & $\begin{array}{l}2.15 \\
2.15\end{array}$ & $\begin{array}{l}12.00 \\
12.00\end{array}$ \\
\hline & & & 2.00 & \\
\hline \multicolumn{5}{|c|}{ BEANS-Pole or Climbing } \\
\hline \multicolumn{4}{|c|}{38 Golden Cluster Wax......... } & \\
\hline & & 1. & & \\
\hline & & & & \\
\hline & & & & \\
\hline & & 1.2 & & \\
\hline & & 1. & & \\
\hline & & & & \\
\hline & & & & \\
\hline & Sea & & 2.50 & \\
\hline \multicolumn{5}{|c|}{ BEANS-Pole Lima } \\
\hline & & & & \\
\hline & & & & \\
\hline & & & & \\
\hline & & & & \\
\hline & Carolina or Sieva.. & & 2.15 & \\
\hline \multicolumn{5}{|c|}{ BEANS-Bush Lima } \\
\hline & & & & \\
\hline & & & & \\
\hline & & & & \\
\hline & & & & \\
\hline & Burpee's & & & \\
\hline
\end{tabular}

\section{Seeds Delivered by Parcel Post or Express Free} of all Charges Except Where Noted

All seeds offered in any quantity listed postpaid or express prepaid in this Special Price List will be delivered to your home, post office, or express office free of all charges.

$100 \mathrm{lb}$. lots of Beans, Sweet Corn, Peas, Mushroom Spawn, Onion Sets, Potatoes, Bulbs and Roots, listed not prepaid. Customers pay transportation charges on these goods.

NOTE-All Beans you may order 2 lbs. or over at 5 lb. rate; 6 lbs, or over at $10 \mathrm{lb}$. rate, postpaid or express prepaid; $15 \mathrm{lbs}$. or over at $100 \mathrm{lb}$. rate, not prepaid. 


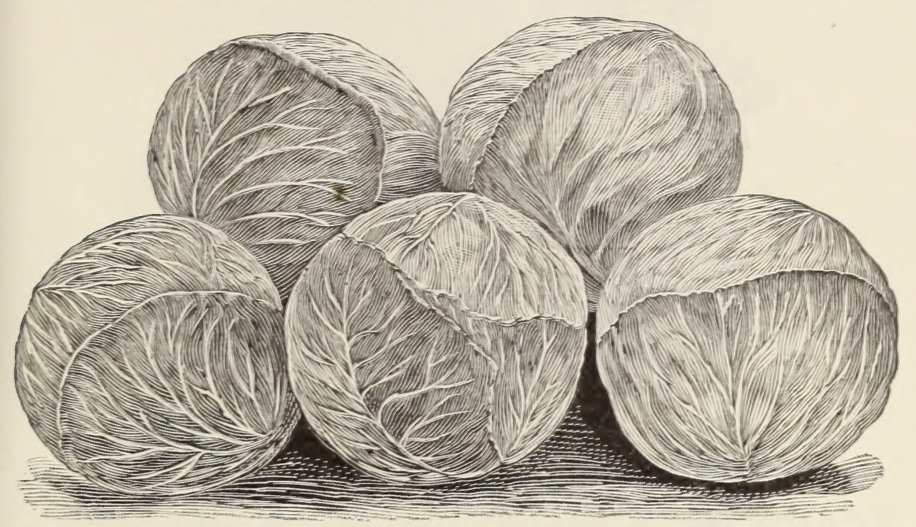

110 Golden Acre Cabbage

\section{CABBAGE-Extra Early Varieties}

Postpaid or Express Prepaid

$1 \mathrm{oz} .1 / 4 \mathrm{lb} .1 \mathrm{lb} .5 \mathrm{lbs} .10 \mathrm{lbs}$.

110 Golden Acre.......... \$0.35 $\$ 1.10 \$ 3.50 \$ 15.75 \$ 30.00$

111 Maule's First Early. ... $\quad .35 \quad 1.10 \quad 3.90 \quad 17.00 \quad 32.00$

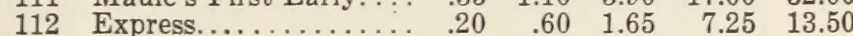

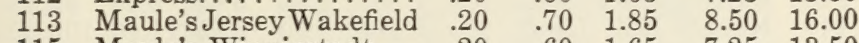

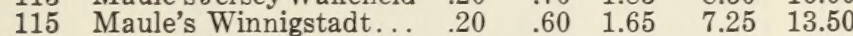

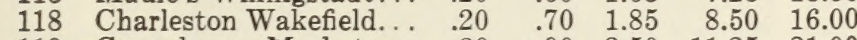

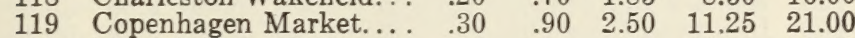

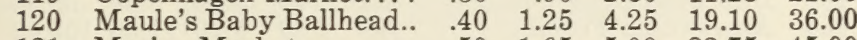

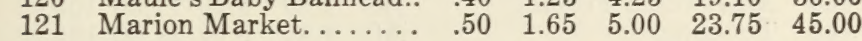

\section{Second Early or Summer Varieties}

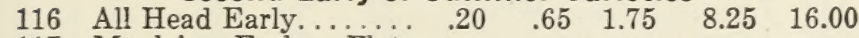

117 Maule's Early Flat

$\begin{array}{llllll}\text { Dutch.............. } & .20 & .60 & 1.65 & 7.25 & 13.50\end{array}$

126

127

Maule's Midsummer...

Glory of Enkhuizen....

Wisconsin All Seasons.

Succession.............. .20

$.70 \quad 1.90$

$8.50 \quad 16.00$

$\begin{array}{llll}.60 & 1.65 & 7.25 & 13.50\end{array}$

132

138

Volga or Stonehead. . . . . 20

$1.10 \quad 3.25$

$.60 \quad 1.65$

$14.60 \quad 28.00$

$\begin{array}{ll}7.25 & 13.50\end{array}$

\section{Late or Winter Varieties \\ 40.35 .50}

122

123

124

125

129

130

131

133

134

135

140

141

Penn State Ball Head. .

Wisconsin Hollander...

Danish Round Head S.S.

Danish Ball Head M.S.

Chihli, Chinese........

Pe-Tsai, Chinese........ . . 20

Maule's Genuine Surehead.................

Maule's Prize Drumhead Maule's Prize Flat Dutch

The Houser............. .35

Red Danish Round Head
Improved Drumhead

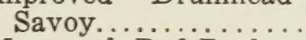

143 $\begin{array}{lll}.35 & 1.20 & 4.00\end{array}$

$.85 \quad 2.25$

$.85 \quad 2.25$

$.80 \quad 2.00$

$\begin{array}{ll}.60 & 1.50\end{array}$

$.65 \quad 1.75$

$.60 \quad 1.65$

$.60 \quad 1.65$

$1.15 \quad 3.90$

.902 .50

$\begin{array}{lll}.25 & .75 & 2.00\end{array}$

$20.25 \quad 39.00$

$18.00 \quad 35.00$

$10.10 \quad 18.00$

$10.10 \quad 18.00$

$8.75 \quad 16.00$

$7.00 \quad 13.00$

$8.25 \quad 16.00$

$7.25 \quad 13.50$

$\begin{array}{ll}7.25 & 13.50\end{array}$

$17.55 \quad 32.00$

$\begin{array}{ll}11.25 & 22.00\end{array}$

\section{$9.00 \quad 16.00$}

8.7516 .00

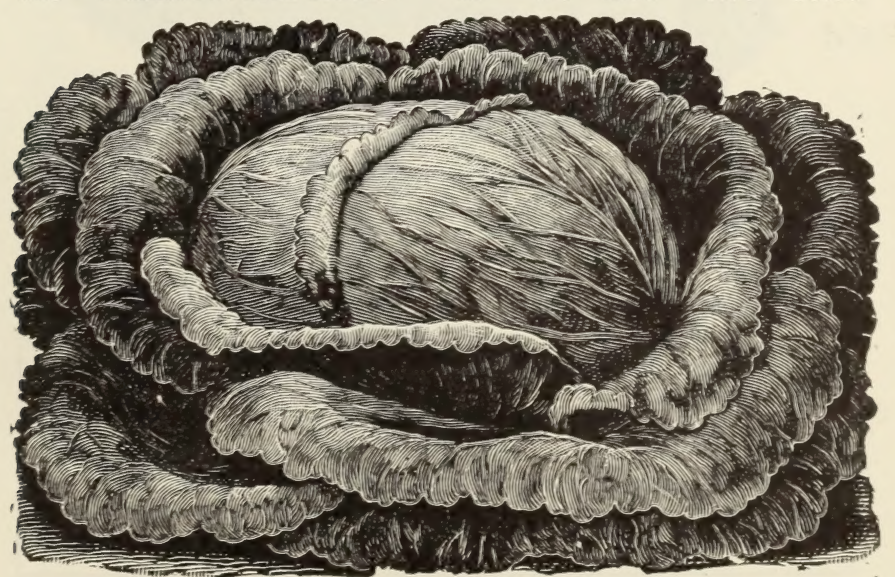

111 Maule's First Early Cabbage

NOTE-All Seeds you may order half lbs. at lb. rate; 2 lbs. or over at 5 lb. rate; 6 lbs. or over at $10 \mathrm{lb}$. rate, postpaid or express prepaid. 


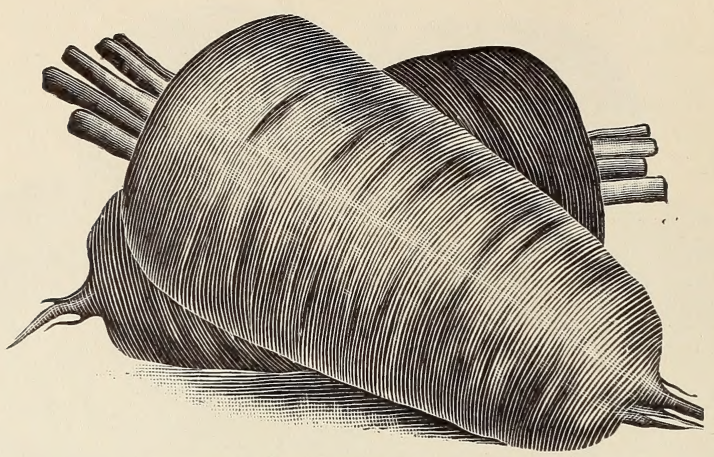

\section{CARROTS}

151 Chantenay or Model Carrot

\begin{tabular}{|c|c|c|c|c|c|c|c|}
\hline & & $\$ 0.45$ & & & & & \\
\hline & Maule's Golden Rod & .45 & 1.10 & & & & \\
\hline & Oxheart or Guerande. & .35 & .80 & & & & \\
\hline & e's Goldinhart & .50 & 1.30 & & & 11.00 & 100 \\
\hline & Horn. & .40 & 1.05 & & & & \\
\hline & Half Long. & .50 & 1.30 & & & 11. & \\
\hline & & .40 & & & & & \\
\hline & & & & & & & \\
\hline & Drange. & .25 & .70 & & & & \\
\hline & & .50 & 1.30 & & & & \\
\hline & & .5 & 1.30 & & & & \\
\hline & & & .75 & & & & \\
\hline & & .2 & .70 & & & & \\
\hline & Large Wh & .2 & .70 & & & & \\
\hline & Large Yel & & & & & & \\
\hline
\end{tabular}

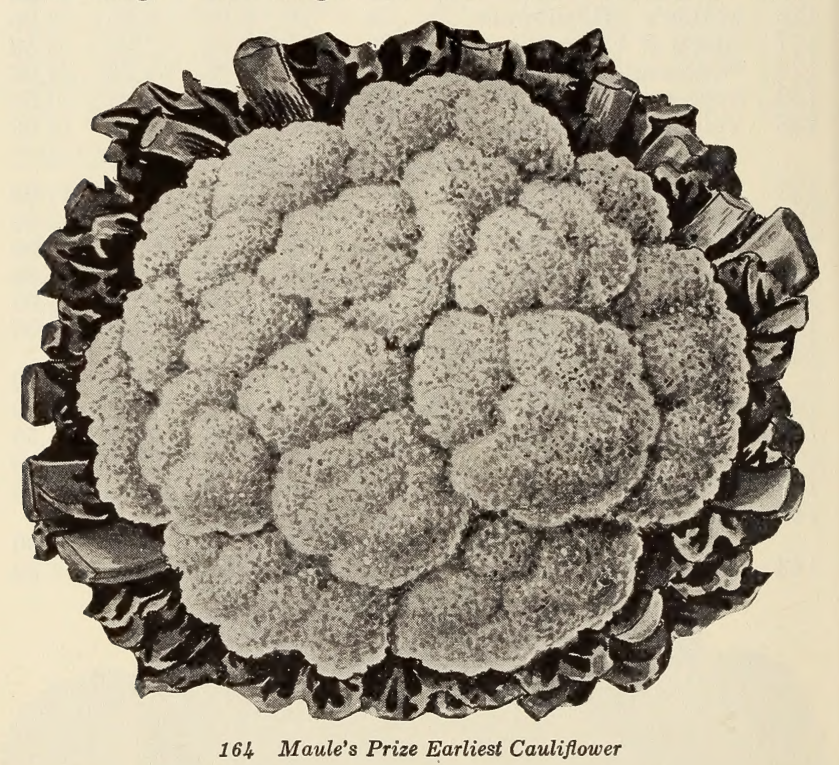

CAULIFLOWER

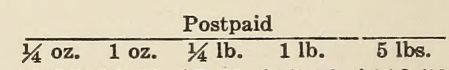

164 Maule's Prize Earliest. $\$ 0.85 \$ 2.50 \$ 7.50 \$ 25.00 \$ 112.50$

165 Dry Weather........ $\quad .70 \quad 2.20 \quad 6.60 \quad 22.00 \quad 100.00$

$\begin{array}{lllllll}168 & \text { Earliest Snowball..... } & .70 & 2.20 & 6.60 & 22.00 & 100.00\end{array}$

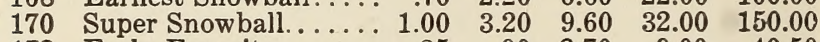

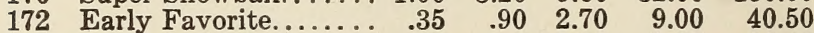

173 Autumn Giant........

REMEMBER WE MAKE NO CHARGE FOR

NOTE-All seeds you may order half lbs. at lb. rate; 2 lbs, or over at $5 \mathrm{lb}$. rate; $6 \mathrm{lbs}$. or over at $10 \mathrm{lb}$ rate; $15 \mathrm{lbs}$. or over at $100 \mathrm{lb}$. rate, postpaid or express prepaid. 


\section{CELERY}

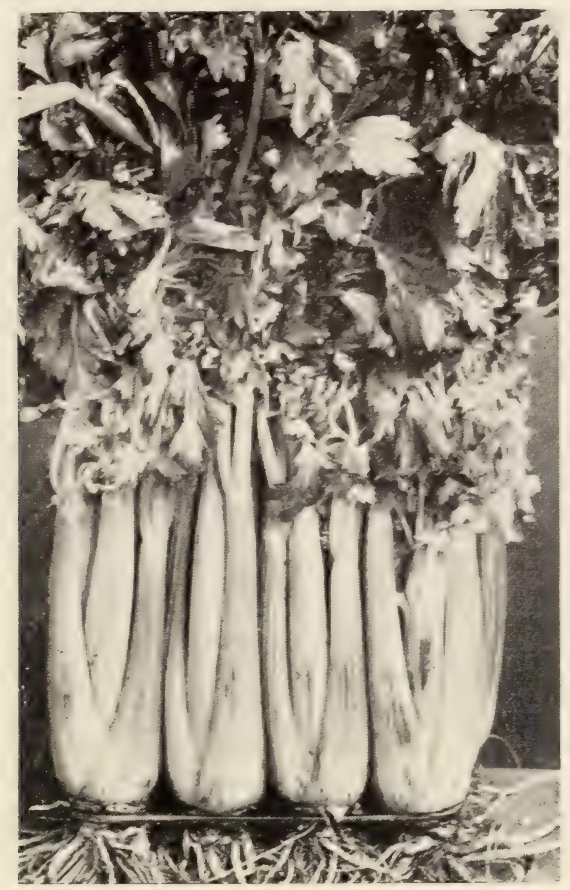

176 Golden Plume or Wonderful Celery

175 Golden S elf

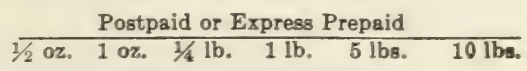
Blanching,

Dwarf French.. $\$ 0.50 \$ 0.85 \$ 2.50 \$ 8.50 \$ 41.25 \$ 80.00$

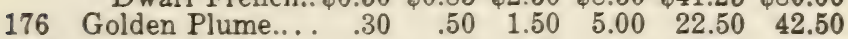

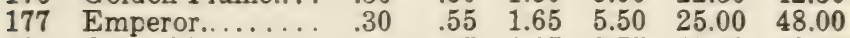

$\begin{array}{lllllll}180 & \text { Columbia........... } & .35 & 1.15 & 3.75 & 17.50 & 32.00\end{array}$

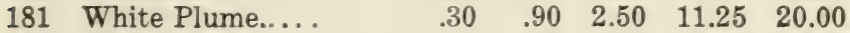

182 Giant Pascal..... $\quad \begin{array}{llllll}18 & .25 & .80 & 2.10 & 10.00 & 17.50\end{array}$

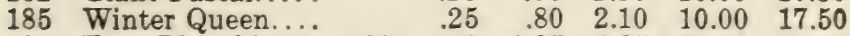

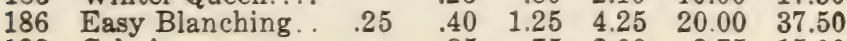

$\begin{array}{lllllll}188 & \text { Celeriac ........ } & .25 & .75 & 2.00 & 8.75 & 15.00\end{array}$

189 American Yellow. $\quad \begin{array}{llllll}35 & 1.20 & 4.00 & 18.75 & 35.00\end{array}$

\section{Order by Number or Name}

Order; all seeds by correct number or by name, but if both are given mistakes are less liable to occur.

\section{CHICORY}

$$
\frac{\text { Postpaid or Express Prepaid }}{1 / 4 \mathrm{lb} .1 \mathrm{lb} .5 \mathrm{lbs} . \quad 10 \mathrm{lbs} .}
$$

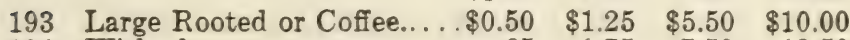

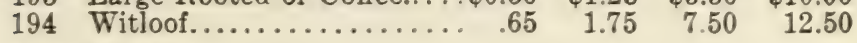

\section{COLLARDS}

201 True Georgia............. $\quad .20 \quad .50 \quad 2.10 \quad 4.00$

\section{CORN SALAD}

203 Large Round-Leaved...... $\quad .40 \quad 1.00 \quad 4.50 \quad 8.00$

\section{CRESS OR PEPPER GRASS}

238 Extra Curled.............. $\quad .20 \quad .60 \quad 2.50 \quad 4.85$

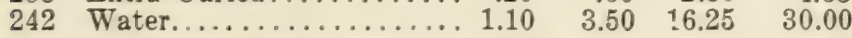

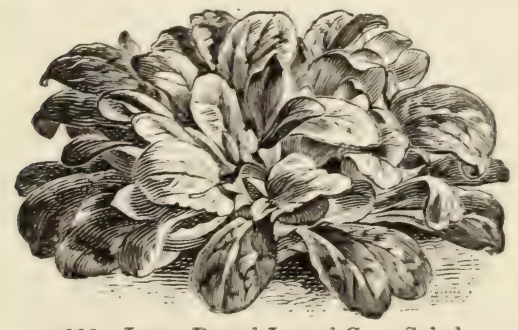

203 Large Round-Leaved Corn Salad

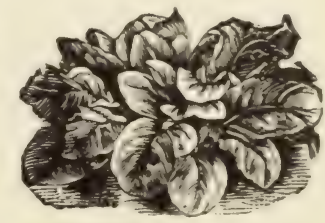

242 Water Cress

NOTE-All seeds you may order half lbs. at lb. rate; 2 lbs. or over at $5 \mathrm{lb}$. rate; $6 \mathrm{lbs}$, or over at $10 \mathrm{lb}$. rate, postpaid or express prepaid. 


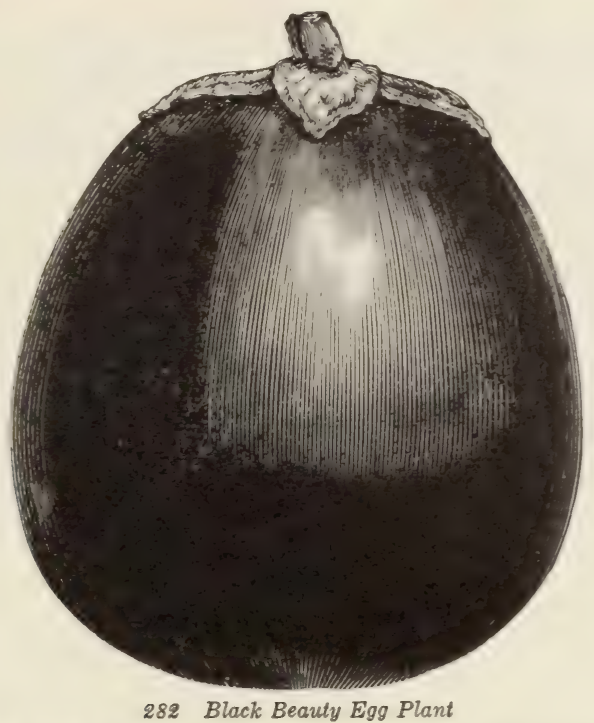

EGG PLANT

282 Black Beauty Egg Plant

$277 / 2$ oz. $1 \mathrm{oz}, 1 / 4 \mathrm{lb} .1 \mathrm{lb} .5 \mathrm{lbs} .10 \mathrm{lbs}$ 277 Maule's Excelsior $\$ 0.25 \quad \$ 0.40 \$ 1.20 \$ 4.00 \$ 18.75 \$ 35.00$ $281 \quad$ N.Y. Imp. Purple $\quad .20 \quad .35 \quad 1.20 \quad 4.00 \quad 18.75 \quad 35.00$

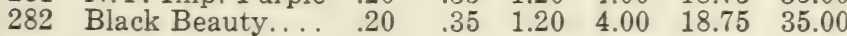

\section{ENDIVE}

$1 / 4 \mathrm{lb}$. $1 \mathrm{lb}$. 5 lbs. $10 \mathrm{lbs}$

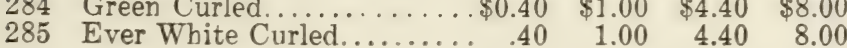

287 Broad Leaved Batavian...... $\quad .40 \quad 1.00 \quad 4.40 \quad 8.00$

\section{B64 GARLIC SETS}

5 lbs., $\$ 1.50$; 10 lbs., $\$ 2.75$ postpaid. 100 lbs., $\$ 18.00$ not

\section{HORSE RADISH SETS} prepaid.

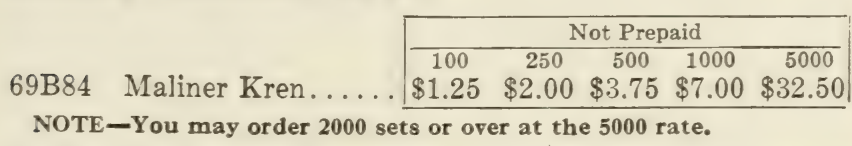

\section{KOHL-RABI}

$10 \mathrm{lb}$. $1 \mathrm{lb}$. $5 \mathrm{lbs} .10 \mathrm{lbs}$

$\begin{array}{lllll}310 & \text { Improved Imperial White. } \$ 0.60 & \$ 1.50 & \$ 6.60 & \$ 12.50\end{array}$

312 Early Purple Vienna...... $\quad .55 \quad 1.50 \quad 6.15 \quad 12.50$

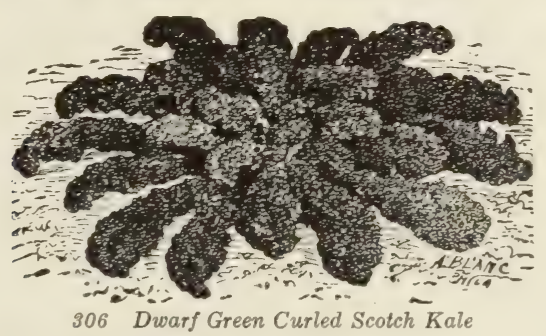

\section{KALE, OR BORECOLE}

Postpaid or Express Prepaid $1 / 4 \mathrm{lb} .1 \mathrm{lb} .5 \mathrm{lbs} .10 \mathrm{lbs} .100 \mathrm{lbs}$.

303 Emerald Isle.......... \$0.35 $\$ 0.90 \$ 4.00 \$ 7.00 \$ 65.00$

304 Dwarf Siberian......... $.20 \quad .60 \quad 2.50 \quad 4.50 \quad 42.50$

306 Dwarf Green Curled

$\begin{array}{llllll}\text { Scotch................ } & .35 & .80 & 3.40 & 6.50 & 62.50\end{array}$

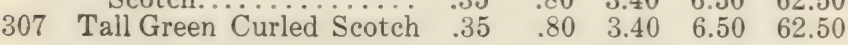

\section{LEEK}

313 Giant Italian. . . . . . . . $50 \quad 1.30 \quad 5.75 \quad 11.00 \quad 100.00$

NOTE-All seeds you may order half lbs. at lb. rate; 2 lbs. or over at $5 \mathrm{lb}$. rate; $6 \mathrm{lbs}$. or over at $10 \mathrm{lb}$. rate; $15 \mathrm{lbs}$. or over at $100 \mathrm{lb}$. rate, postpaid or express prepaid. 


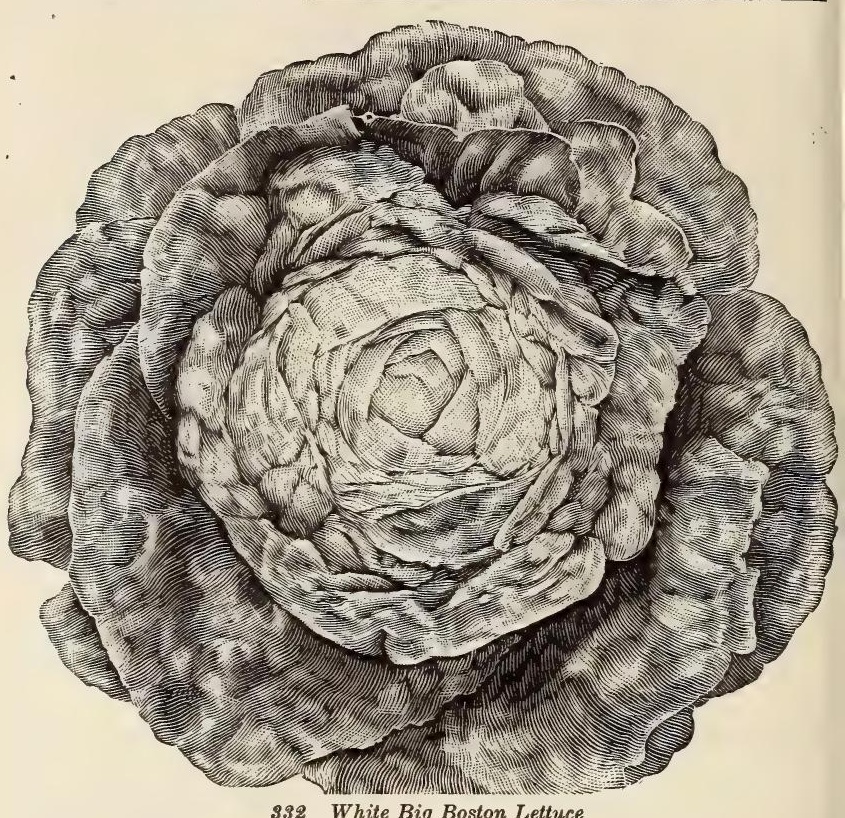

\section{LETTUCE-Heading Varieties}

Postpaid or Express Prepaid

316 Maule's Immensity $1 / 4 \mathrm{lb} .1 \mathrm{lb} .5 \mathrm{lbs} .10 \mathrm{lbs} .100 \mathrm{lbs}$.

318 May King.............40 $\quad .400 \quad 4.50 \quad 8.00 \quad 77.50$

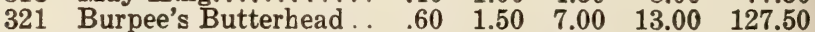

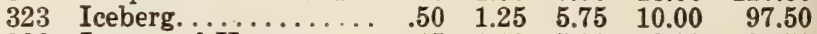

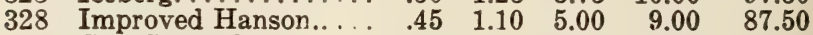

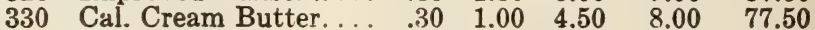

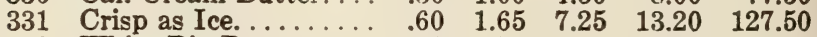

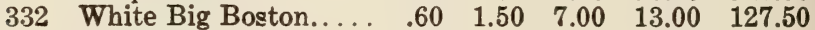

$\begin{array}{lllllll}333 & \text { New York No. } 12 \ldots \ldots & .75 & 2.00 & 8.75 & 16.00 & 150.00\end{array}$

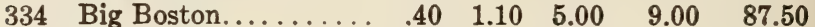

$\begin{array}{lllllll}340 & \text { Earliest Wayahead.... } & .60 & 1.50 & 7.00 & 13.00 & 127.50\end{array}$

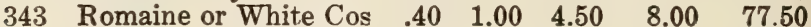

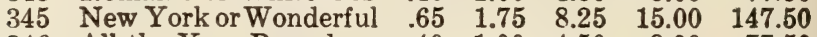

346 All the Year Round... $40 \quad 40 \quad 1.00 \quad 4.50 \quad 8.00 \quad 77.50$

$\begin{array}{lllllll}\text { Loose Leaved or Cutting Varieties } & & \\ 326 & \text { Early Prize Head ..... } & .40 & 1.00 & 4.50 & 8.00 & 77.50\end{array}$

341 Chicken.................40 $\quad .00 \quad 4.50 \quad 8.00 \quad 77.50$

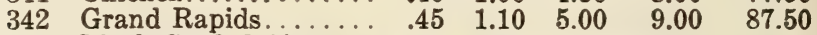

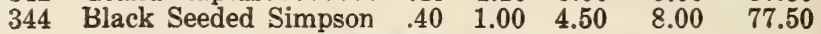

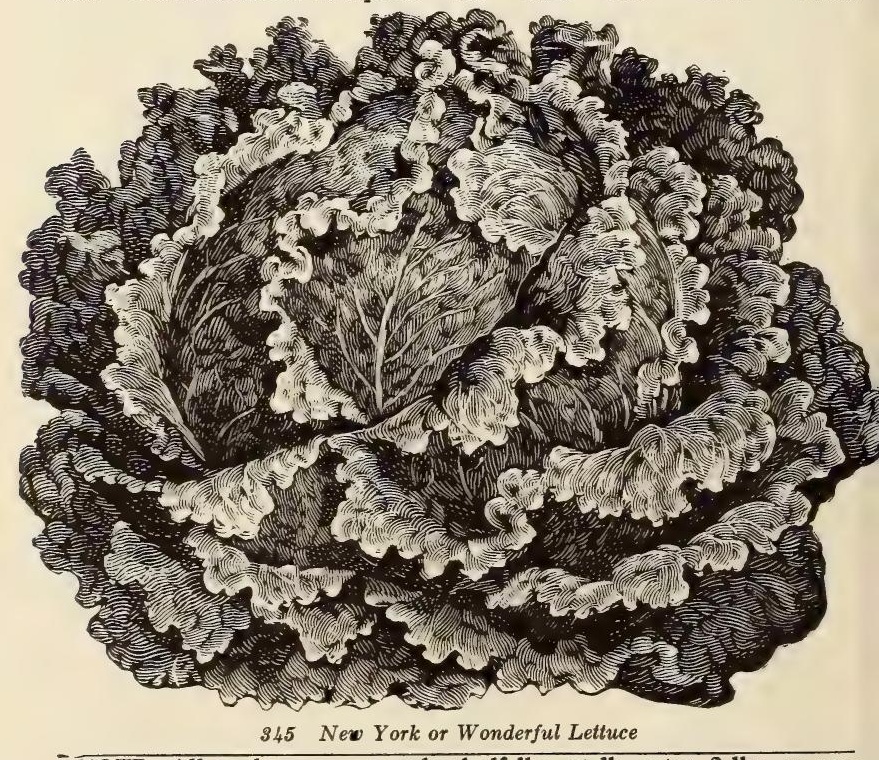

- NOTE-All seeds you may order half lbs, at lb. rate; 2 lbs. or over at $5 \mathrm{lb}$. rate; $6 \mathrm{lbs}$. or over at $10 \mathrm{lb}$. rate; $15 \mathrm{lbs}$. or over at $100 \mathrm{lb}$. rate. postpaid or express prepaid. 
404 Maule's Improved Kleckley Sweet Watermelon

\section{WATERMELON}

387 Panmure All-Heart.

\section{Postpaid or Express Prepaid}

$1 / 4 \mathrm{lb}$. $1 \mathrm{lb} .5 \mathrm{lbs} .10 \mathrm{lbs} .100 \mathrm{lbs}$

All-Heart.. . \$ \$0.55 \$1.35 \$6.25 \$11.50 \$110.00

$\begin{array}{llllll} & & \end{array}$

$\begin{array}{llllll}\text { Excel (Black Seeded). } & .35 & .85 & 3.50 & 6.50 & 60.00\end{array}$

$\begin{array}{llllll}\text { Fordhook Early....... } & .20 & .60 & 2.50 & 4.50 & 42.50\end{array}$

$\begin{array}{llllll}\text { Thurmond Gray....... } & .25 & .70 & 3.00 & 5.50 & 52.50\end{array}$

$\begin{array}{llllll}\text { Kleckley Sweet. ..... } & .25 & .70 & 3.00 & 5.50 & 52.50\end{array}$

$\begin{array}{llllll}\text { Gray Monarch ........ } & .25 & .70 & 3.00 & 5.25 & 50.00\end{array}$

$\begin{array}{llllll}\text { Tom Watson......... } & .25 & .70 & 3.00 & 5.50 & 52.50\end{array}$

$\begin{array}{llllll}\text { Georgia Rattlesnake.. . } & .25 & .70 & 3.00 & 5.25 & 50.00\end{array}$

Harris' Earliest....... $\quad .30 \quad .70 \quad 3.25 \quad 6.00 \quad 55.00$

$\begin{array}{llllll}\text { Maule's National..... } & .25 & .70 & 3.00 & 5.50 & 52.50\end{array}$

$\begin{array}{llllll}\text { Stone Mountain...... } & .35 & .90 & 4.20 & 7.50 & 72.50\end{array}$

Angel's Kiss......... . .25 $\quad .75 \quad 3.50 \quad 6.00 \quad 57.50$

Florida Favorite. .... $.25 \quad .70 \quad 3.25 \quad 6.00 \quad 55.00$

Imp. Kleckley Sweet. . $\quad .35 \quad .85 \quad 3.65 \quad 6.50 \quad 62.50$

$\begin{array}{llllll}\text { Irish Gray............. } & .25 & .70 & 3.00 & 5.50 & \mathbf{5 2 . 5 0}\end{array}$

$\begin{array}{llllll}\text { Peerless or Ice Cream. } & .25 & .70 & 3.00 & 5.25 & 50.00\end{array}$

Golden Sweet........ $\quad .40 \quad 1.00 \quad 4.50 \quad 8.00 \quad 77.50$

$\begin{array}{llllll}\text { Shaker Blue ......... } & .25 & .00 & 3.00 & 5.25 & 50.00\end{array}$

Winter.............. $\quad .45 \quad 1.10 \quad 5.00 \quad 9.00 \quad 87.50$

Phinney's Early....... $.25 \quad .70 \quad 3.00 \quad 5.25 \quad 50.00$

Halbert Honey....... . . .35 $\quad .85 \quad 3.65 \quad 6.50 \quad 62.50$

Baby's Delight...... $.60 \quad 1.50 \quad 7.00 \quad 13.00 \quad 127.50$

$\begin{array}{llllll}\text { Perfection............ } & .40 & .95 & 4.25 & 7.50 & 72.50\end{array}$

$\begin{array}{llllll}\text { Apple Pie Citron Melon } & .25 & .65 & 3.00 & 5.00 & 50.00\end{array}$

$\begin{array}{llllll}\text { Colo. Preserving Citron. } & .35 & .85 & 3.65 & 6.50 & 62.50\end{array}$

\section{OKRA OR GUMBO}

Postpaid or Express Prepaid

131 1/4. 1 lb. 5 lbs. $10 \mathrm{lbs} .100 \mathrm{lbs}$.

431 Perkin's Perf.Long Pod $\$ 0.20 \quad \$ 0.50 \quad \$ 2.25 \quad \$ 4.25 \quad \$ 40.00$

433 Dwarf Georgia Favorite $\begin{array}{lllll}.20 & .55 & 2.25 & 4.25 & 40.00\end{array}$

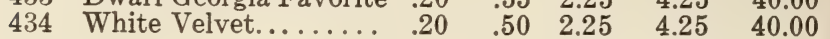

\section{PARSLEY}

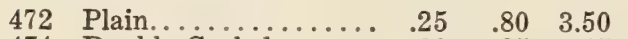

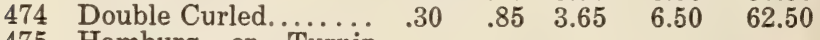

$\begin{array}{rrrrrr}\text { Hamburg } \begin{array}{c}\text { or Turnip } \\ \text { Rooted............. }\end{array} .40 & .95 & 4.40 & 7.50 & 72.50\end{array}$

\section{PARSNIP}

477 Maule's White Lisbon.

478 Maule's Improved Half Long. .................

483 Long Smooth or Hollow Crown..............

$\begin{array}{lllll}.25 & .75 & 3.50 & 6.00 & 57.50 \\ .25 & .70 & 3.00 & 5.25 & 50.00 \\ .20 & .60 & 2.50 & 4.75 & 45.00\end{array}$

\section{MUSHROOM SPAWN}

69B68 Pure Culture Bricks

Not Prepaid: 5 bricks, $\$ 1.15$; 10 bricks, $\$ 2.20$; 25 bricks, $\$ 5.25 ; 100$ bricks, $\$ 20.00$.

\section{B66 Pure Spore Culture}

Not Prepaid: 1 carton, $\$ 1.00 ; 5$ cartons, $\$ 4.50 ; 10$ cartons, $\$ 8.00$.

NOTE-All seeds you may order half lbs. at lb. rate; 2 lbs, or over at $5 \mathrm{lb}$. rate; $6 \mathrm{lbs}$. or over at $10 \mathrm{lb}$. rate; $15 \mathrm{lbs}$. or over at $100 \mathrm{lb}$. rate, postpaid or express prepaid. 


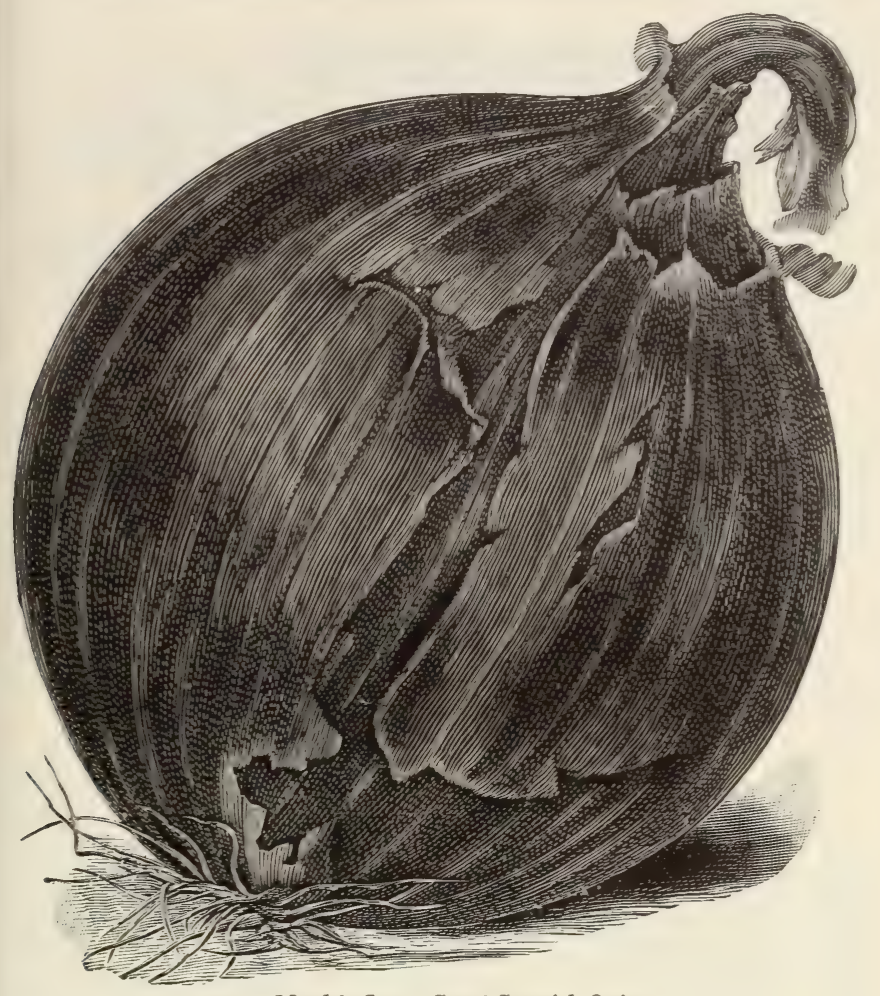

439 Maule's Large Sweet Spanish Onion

ONION

\begin{tabular}{l} 
Postpaid or Express Prepaid \\
\hline $1 / 4 \mathrm{lb} .1 \mathrm{lb} .5 \mathrm{lbs} . \quad 10 \mathrm{lbs} . \quad 100 \mathrm{lbs}$.
\end{tabular}

436 Large Red Wethersfield $\$ 0.50 \$ 1.30 \$ 6.00 \$ 11.00 \$ 105.00$

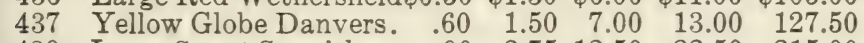

$439 \quad$ Large Sweet Spanish. . . $\quad .90 \quad 2.75 \quad 12.50 \quad 22.50 \quad 215.00$

441 Maule's Prizetaker.... $\quad .65 \quad 1.75 \quad 8.25 \quad 15.00 \quad 145.00$

442 White Welsh........ $.65 \quad 1.75 \quad 8.25 \quad 15.00 \quad 145.00$

444 Southport Red Giobe. $\quad .60 \quad 1.50 \quad 7.00 \quad 13.00 \quad 127.50$

445 Southport White Globe $\quad .80 \quad 2.25 \quad 10.50 \quad 20.00 \quad 195.00$

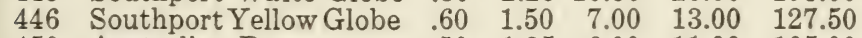

450 Australian Brown..... $.50 \quad 1.35 \quad 6.00 \quad 11.00 \quad 105.00$

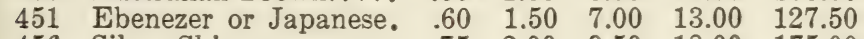

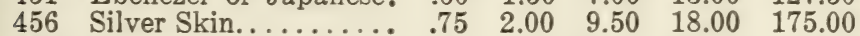

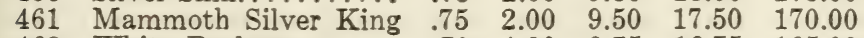

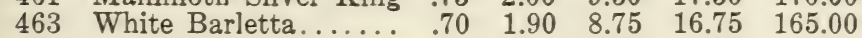

$\begin{array}{lllllll}466 & \text { Extra Early Pearl.... } & .65 & 1.75 & 8.25 & 16.00 & 155.00\end{array}$

\section{ONION SETS}

69B70 Yellow Globe Danvers. ...... \$0.95 $\$ 1.75 \quad \$ 9.00$

69B72 White Silver Skin........... $1.00 \quad 1.85 \quad 10.00$

69B74 Large Red Wethersfield .......

69B76 Yellow Ebenezer........... $.95 \quad 1.75 \quad 9.00$

\section{POTATOES}

Maine Grown Certified Stock

69B90 Irish Cobbler.

Burpee's Extra Early... ..... $1.85 \quad 4.25 \quad$ |20.00

69B92 Pure Early Rose........... $1.85 \quad 4.25 \quad 20.00$

69B94 Maule' Early Thoroughbred. $1.85 \quad 4.25 \quad 20.00$

69B96 The Snow.................. $1.85 \quad 4.25 \quad 20.00$

69B98 Green Mountain 'State of Maine)................ $1.75 \quad 4.00 \quad 19.25$ Date of Shipment Potatoes will be shipped at any date are given to the contrary, we will hold same until in our judgment there will be no danger of freezing, then ship promptly.

NOTE-All seeds you may order half 1 bs. at $1 \mathrm{~b}$. rate; 2 lbs. or over at 5 lb. rate; $6 \mathrm{lbs}$. or over at $10 \mathrm{lb}$. rate; $15 \mathrm{lbs}$. or over at $100 \mathrm{lb}$. rate, postpaid"or"express prepaid. Potatoes 2 or more bbl. sacks at 5 bbl. sacks rate, not prepaid. 


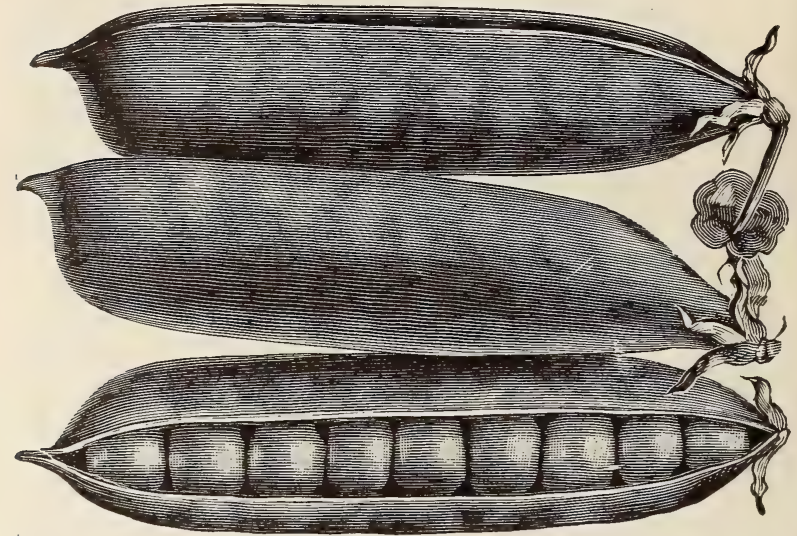

487 Early Bird Pea

\section{PEAS-Smooth Extra Early Varieties}

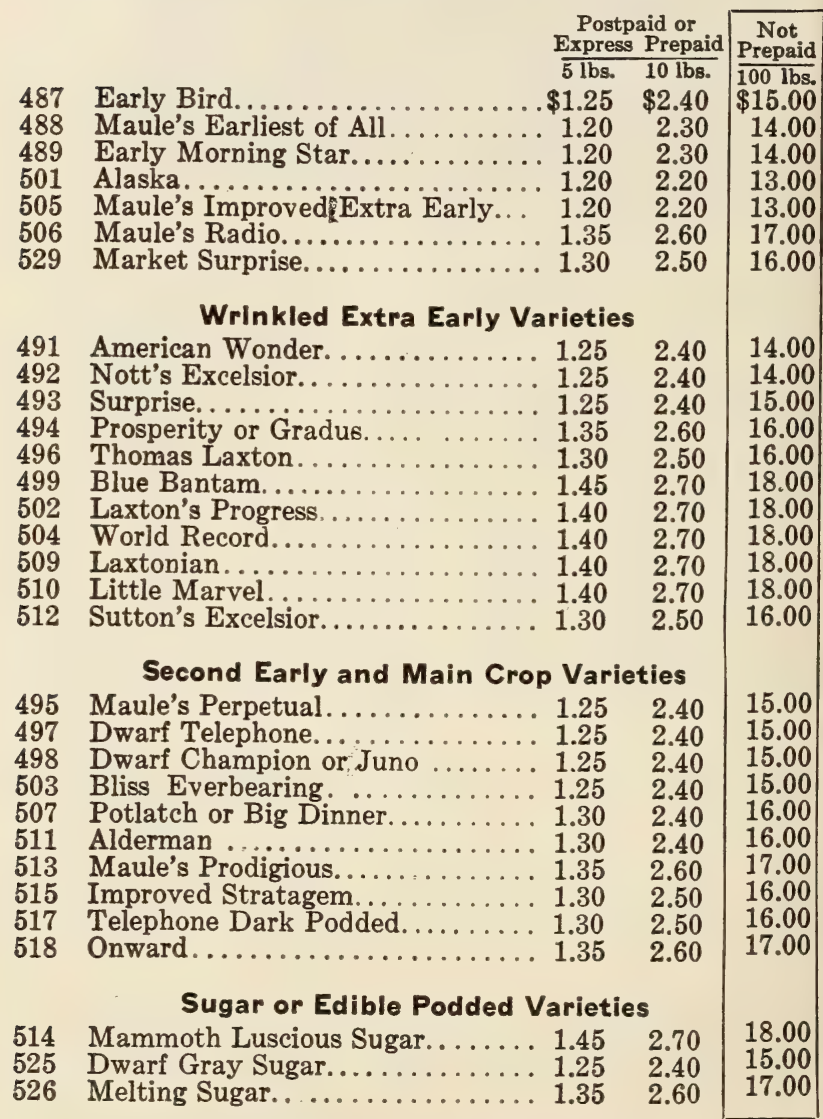

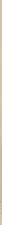

507 Potlatch or Big Dinner Pea

NOTE-All peas you may order 2 lbs. or over at $5 \mathrm{lb}$, rate; $6 \mathrm{lbs}$ or over at $10 \mathrm{lb}$. rate, postpaid, or express prepaid; $15 \mathrm{lbs}$. or over at $100 \mathrm{lb}$. rate, not prepaid. 


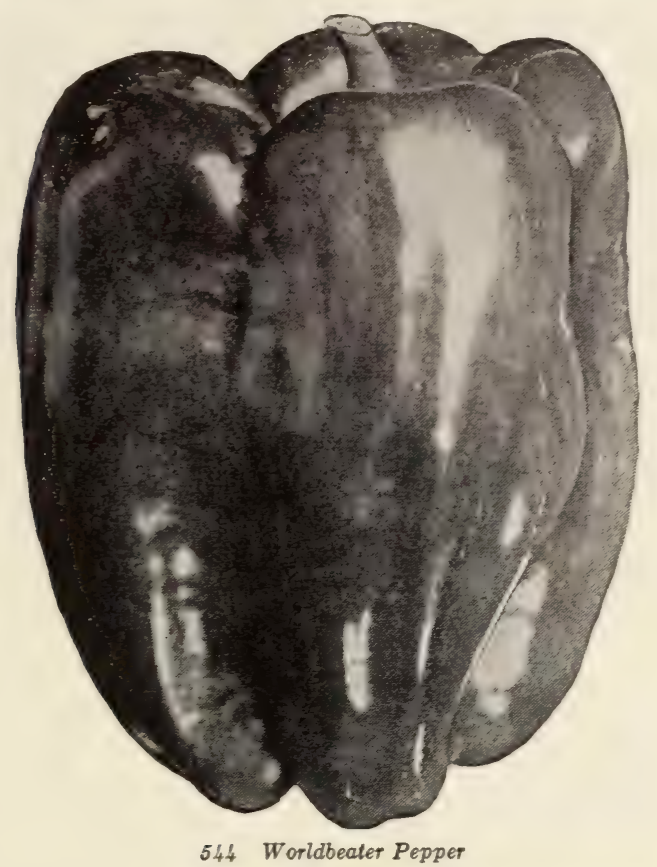

PEPPERS

Postpaid or Express Prepaid

$1 / 2$ oz, $1 \mathrm{oz}, 1 / 4 \mathrm{lb}, 1 \mathrm{lb}, 5 \mathrm{lbs}, 10 \mathrm{lbs}$.

530 Giant Crimson... $\$ 0.25 \$ 0.40 \$ 1.35 \$ 4.50 \$ 21.25 \$ 40.00$

531 Maule's Neapoli-

$\begin{array}{lllllll}\tan \ldots \ldots \ldots \ldots & .20 & .35 & 1.10 & 3.15 & 14.50 & 27.50\end{array}$

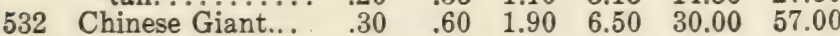

533 Improved Ruby

$\begin{array}{lllllll}\text { King .......... } & .20 & .30 & .95 & 2.90 & 13.00 & 25.00\end{array}$

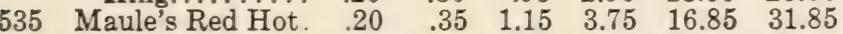

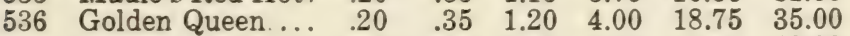

$\begin{array}{llllllll}537 & \text { Tobasco } \ldots \ldots \ldots & .25 & .40 & 1.35 & 4.50 & 21.25 & 40.00\end{array}$

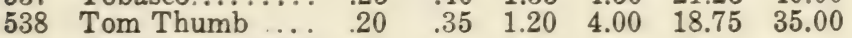

539 Maule's Early

$\begin{array}{lllllll}\text { Giant......... } & .25 & .45 & 1.40 & 4.90 & 22.00 & 42.00\end{array}$

$\begin{array}{llllllll}540 & \text { Pimento.... } & .15 & .25 & 85 & 2.40 & 11.00 & 20.00\end{array}$

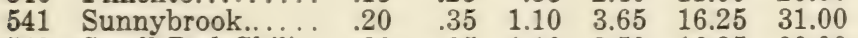

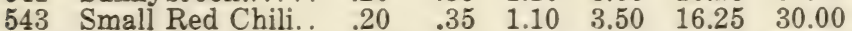

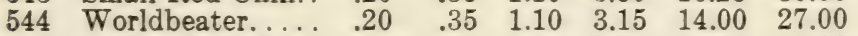

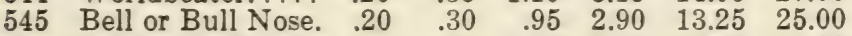

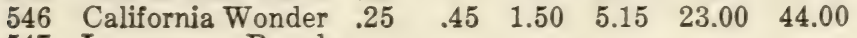

$547 \quad$ L o n g $R$ e d

$\begin{array}{lllllll}\text { Cayenne....... } & .20 & .35 & 1.10 & 3.50 & 16.25 & 30.00\end{array}$

\section{Order by Number or Name}

Order all seeds by correct number or by name, but if both are given mistakes are less liable to occur.

\section{PUMPKIN}

554 Yellow Sweet Potato Pie... $\$ 0.50 \quad \$ 1.25 \quad \$ 5.75 \quad \$ 10.00$

555 Small Sugar............. . . 25 $\quad .70 \quad 3.00 \quad 5.00$

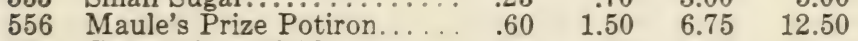

557 Connecticut Field ... 20

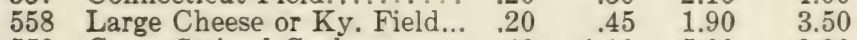

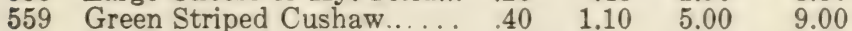

561 Maule's Hundred Weight... $\quad \begin{array}{llll}.95 & 2.75 & 12.35 & 23.35\end{array}$

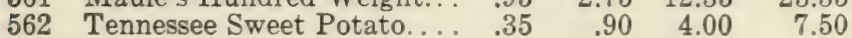

563 Golden Cushaw or Crook-

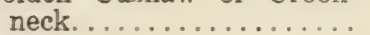

$\begin{array}{llllll}564 & \text { Japanese Pie. . . . . . . . . . . } & .30 & .90 & 4.00 & 7.50 \\ 566 & \text { Winter Luxury or Pie. . . . . } & .40 & .95 & 4.25 & 7.50\end{array}$

REMEMBER WE MAKE NO CHARGE FOR

NOTE-All seeds you may order half $1 \mathrm{bs}$. at lb. rate; 2 lbs. or over at $5 \mathrm{lb}$. rate; $6 \mathrm{lbs}$. or over at $10 \mathrm{lb}$. rate, postpaid or express prepaid. 


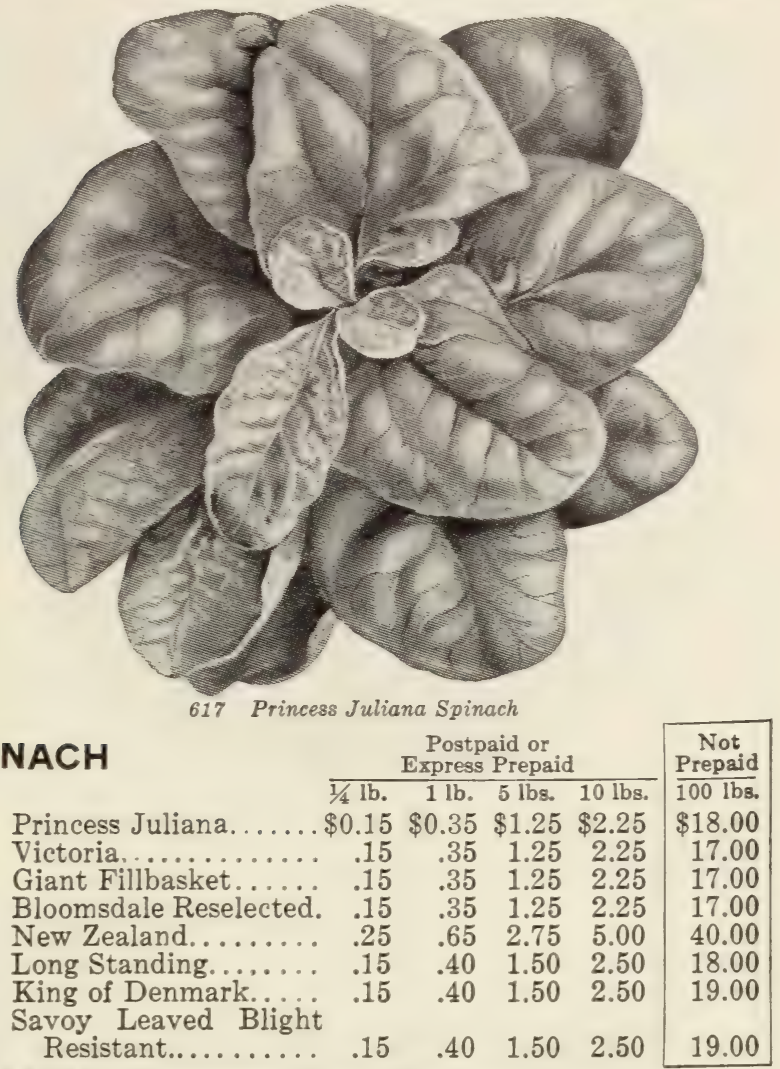

\section{SPINACH}

\section{Postpaid or}

$\begin{array}{llrrrrr}617 & \text { Princess Juliana........ } \$ 0.15 & \$ 0.35 & \$ 1.25 & \$ 2.25 & \$ 18.00 \\ 618 & \text { Victoria.............. } & .15 & .35 & 1.25 & 2.25 & 17.00 \\ 620 & \text { Giant Fillbasket...... } & .15 & .35 & 1.25 & 2.25 & 17.00 \\ 621 & \text { Bloomsdale Reselected. } & .15 & .35 & 1.25 & 2.25 & 17.00 \\ 622 & \text { New Zealand.......... } & .25 & .65 & 2.75 & 5.00 & 40.00 \\ 623 & \text { Long Standing........ } & .15 & .40 & 1.50 & 2.50 & 18.00 \\ 624 & \text { King of Denmark..... } & .15 & .40 & 1.50 & 2.50 & 19.00 \\ 626 & \text { Savoy Leaved Blight } & & & & & \\ & \text { Resistant............ } & .15 & .40 & 1.50 & 2.50 & 19.00\end{array}$

\section{SQUASH-Summer Varieties}

Postpaid or Express Prepaid

1/4 lb. 1 lb. 5 lbs. $10 \mathrm{lbs} .100 \mathrm{lbs}$.

Earlest White Bush... \$0.35 $\$ 0.85 \quad \$ 3.65 \quad \$ 6.50 \quad \$ 62.50$

629 Straightneck Summer. $\quad .50 \quad 1.25 \quad 5.75 \quad 11.00 \quad 100.00$

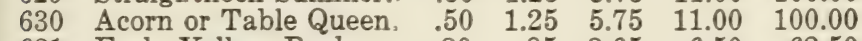

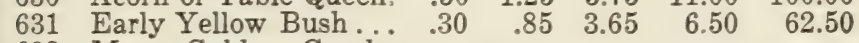

632 Mam. Golden Croolneck............... $.40 \quad 1.00 \quad 4.50 \quad 8.25 \quad 80.00$

639 Cocozelle Bush....... $\quad .40 \quad 1.00 \quad 4.50 \quad 8.50 \quad 82.50$

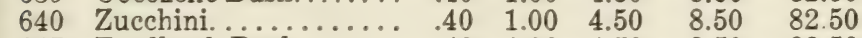

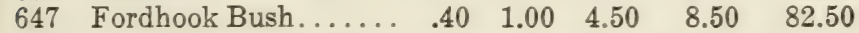

\section{Autumn and Winter Varieties}

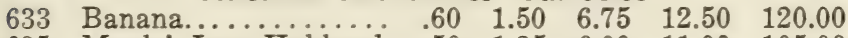

635 Maule's Imp. Hubbard. $\quad .50 \quad 1.35 \quad 6.00 \quad 11.00 \quad 105.00$

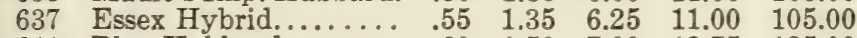

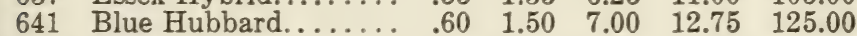

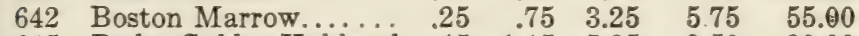

645 Red or Golden Hubbard $\quad \begin{array}{lllll}.45 & 1.15 & 5.25 & 9.50 & 90.00\end{array}$
648
Chi. Warted Hubbard
$\begin{array}{lll}50 & 1.35 & 6.00\end{array}$
11.00
105.00

\section{SALSIFY OR OYSTER PLANT}

614 Mam. Sandwich Island. $\begin{array}{llllll}.75 & 2.00 & 8.75 & 16.50 & 160.00\end{array}$

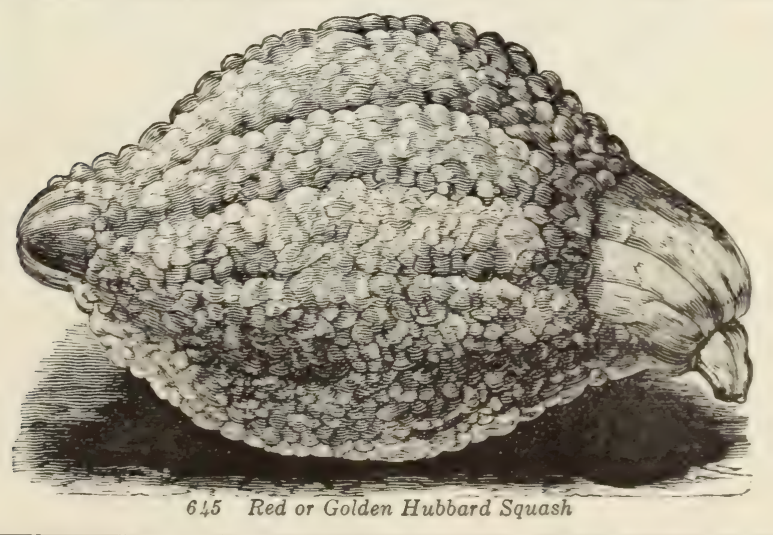

NOTE-All seeds you may order half lbs. at lb. rate; 2 lbs. or over at $5 \mathrm{lb}$. rate; $6 \mathrm{lbs}$. or over at $10 \mathrm{lb}$. rate; $15 \mathrm{lbs}$. or over at $100 \mathrm{lb}$. rate, postpaid or express prepaid. 


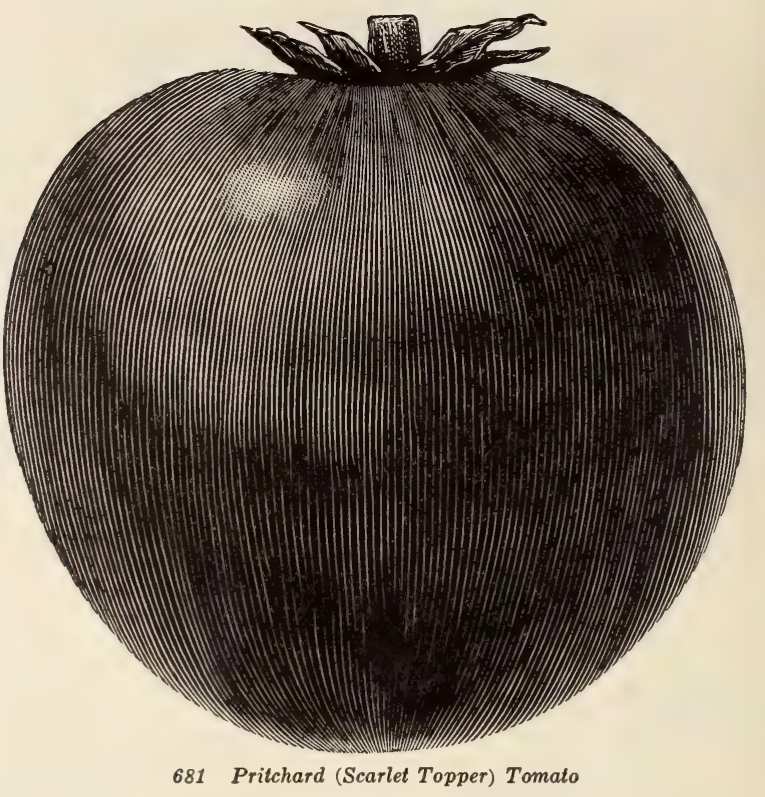

TOMATO-Red Varieties

Postpaid or Express Prepaid

$1 / 2$ oz. 1 oz. $1 / 4 \mathrm{lb}$. $1 \mathrm{lb}$. $5 \mathrm{lbs}$. $10 \mathrm{lbs}$.

651 Earliest of All... $\$ 0.25 \$ 0.40 \$ 1.25 \$ 4.25 \$ 20.00 \$ 37.50$

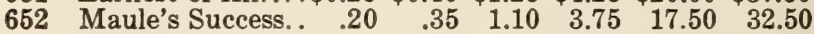

655 Red Head....... $\quad .20 \quad .35 \quad 1.10 \quad 3.75 \quad 17.50 \quad 32.50$

656 Enormous....... . .25 $\quad .45 \quad 1.35 \quad 4.50 \quad 21.25 \quad 40.00$

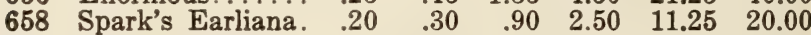

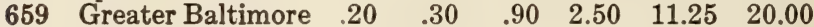

660 John Baer....... $.20 \quad .30 \quad .90 \quad 2.50 \quad 11.25 \quad 20.00$

661 Break O'Day..... $\quad .20 \quad .35 \quad 1.10 \quad 3.50 \quad 16.25 \quad 30.00$

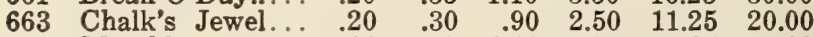

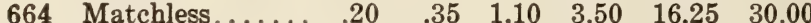

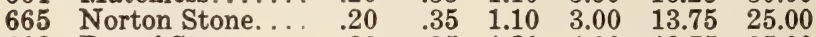

666 Dwarf Stone. . . . . 20 $\quad .35 \quad 1.20 \quad 4.00 \quad 18.75 \quad 35.00$

669 Marglobe........ $20 \quad .35 \quad 1.10 \quad 3.00 \quad 13.75 \quad 25.00$

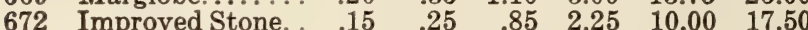

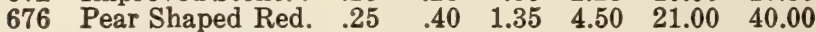

680 Columbia........ $.25 \quad .40 \quad 1.25 \quad 4.00 \quad 18.75 \quad 35.00$

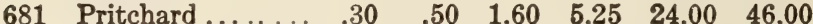

683 Bonny Best Early $\quad .20 \quad .30 \quad \begin{array}{lllll}20 & .90 & 2.50 & 11.25 & 20.00\end{array}$

\section{Pink or Purple Varieties}

657 Maule's Imperial. .25

662 June Pink...... .20

667 Cooper's Special. .25

668 Gulf State Market .20

670 Redfield Beauty.. .20

671

673

678

679

682

Oxheart........45

Dwarf Giant.... . .35

Dwarf Champion. .20

Livingston's Globe .20

$\begin{array}{lllll}.40 & 1.30 & 4.00 & 18.75 & 35.00\end{array}$

$\begin{array}{lllll}35 & 1.10 & 3.65 & 16.00 & 31.00\end{array}$

$\begin{array}{lllll}.40 & 1.30 & 4.25 & 20.00 & 38.00\end{array}$

$\begin{array}{lllll}35 & 1.10 & 3.25 & 15.00 & 27.50\end{array}$

$\begin{array}{lllll}.35 & 1.10 & 3.50 & 16.25 & 30.00\end{array}$

$\begin{array}{lllll}80 & 2.75 & 9.00 & 42.50 & 80.00\end{array}$

$\begin{array}{lllll}65 & 2.10 & 7.00 & 32.00 & 61.00\end{array}$

$\begin{array}{lllll}.65 & 2.10 & 7.00 & 32.00 & 61.00 \\ .35 & 1.20 & 4.00 & 18.75 & 35.00\end{array}$

$\begin{array}{lllll}.35 & 1.10 & 3.15 & 14.00 & 27.00\end{array}$

$\begin{array}{lllllll}\text { Ponderosa....... } & .30 & .50 & 1.65 & 5.50 & 25.00 & 48.00\end{array}$

\section{Yellow Varieties}

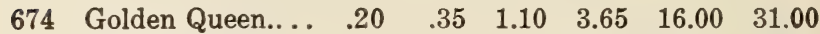

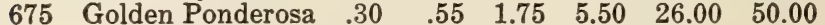

677 Pear S h a p ed $\quad 25 \quad 40 \quad 135 \quad 4.50 \quad 21.00 \quad 40.00$ 684 Tangerine......

\section{HERBS}

Postpaid or Express Prepaid

750 Dill 1 oz. $1 / 4 \mathrm{lb} .1 \mathrm{lb} .4 \mathrm{lbs} .10 \mathrm{lbs}$.

752 Fennel, Sweet........ $.15 \quad .45 \quad 1.20 \quad 5.75 \quad 11.00$

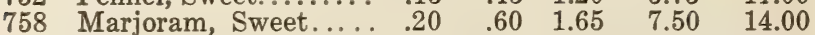

770 Sage.............. .25 $.75 \quad 2.00 \quad 9.00 \quad 17.50$

772 Summer Savory....... $\quad .25 \quad .60 \quad 1.50 \quad 6.60 \quad 12.00$

778 Thyme.................... $35 \quad 1.00 \quad 3.50 \quad 17.00 \quad 32.50$

NOTE-All seeds you may order half 1 bs. at $1 \mathrm{~b}$. rate; 2 lbs. or over at $5 \mathrm{lb}$. rate; $6 \mathrm{lbs}$. or over at $10 \mathrm{lb}$. rate, postpaid or express prepaid. 


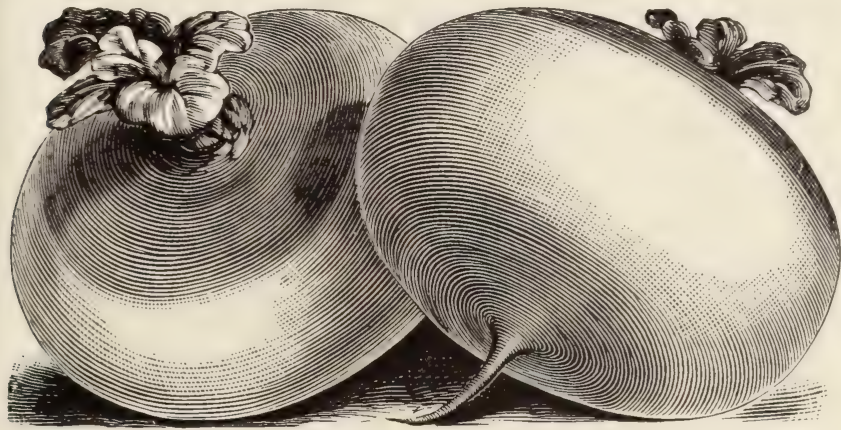

704 Japanese (Shogoin) Turnip

\section{TURNIP}

686 Red Top White Globe.. $\$ 0.20 \$ 0.42 \$ 1.90 \$ 3.65 \$ 35.00$

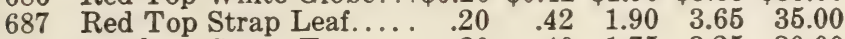

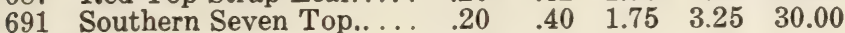

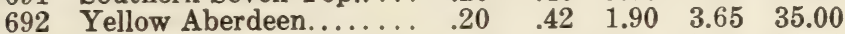

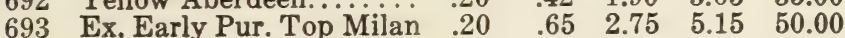

694 Extra Early White Milan 20

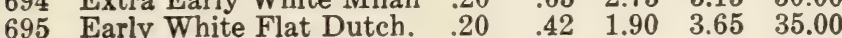

$\begin{array}{llll}-4 & 1.90 & 3.65 & 35.00\end{array}$

697 Cow Horn............. .20 $.42 \quad 1.90 \quad 3.65 \quad 35.00$

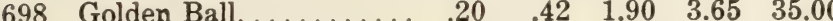

700 White Norfolk Globe.... $\quad .20 \quad .42 \quad 1.90 \quad 3.65 \quad 35.00$

701 White Egg. . $\quad \begin{array}{lllll}20 & 42 & 1.90 & 3.65 & 35.00\end{array}$

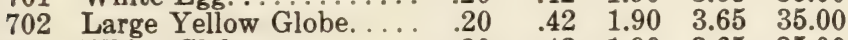

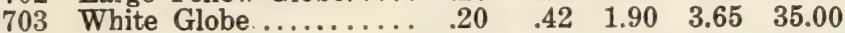

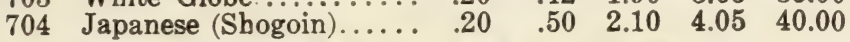

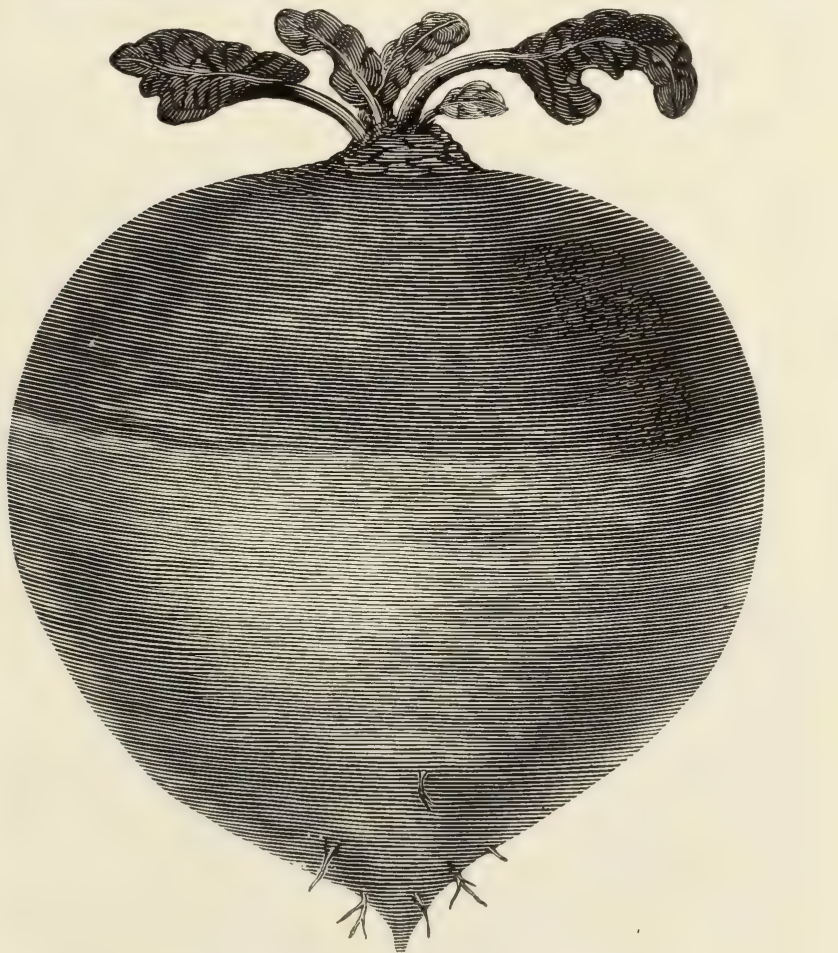

707 Maule's Heavy Cropping Ruta Baga

\section{RUTA BAGA OR SWEDE TURNIP}

Postpaid or Express Prepaid

$1 / 4 \mathrm{lb}, 1 \mathrm{lb}, 5 \mathrm{lbs}, 10 \mathrm{lbs}, 100 \mathrm{lbs}$

707 Maule's Heavy Cropping. $\$ 0.25 \$ 0.65 \$ 3.00 \$ 5.50 \$ 52.50$

$\begin{array}{lllllll}708 & \text { Improved Purple Top } & 20 & .55 & 2.30 & 4.40 & 42.50\end{array}$

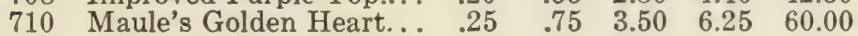

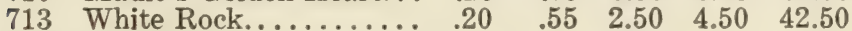

NOTE-All seeds you may order half lbs, at lb. rate; 2 lbs. or over at $5 \mathrm{lb}$. rate; $6 \mathrm{lbs}$. or over at $10 \mathrm{lb}$. rate; $15 \mathrm{lbs}$. or over at $100 \mathrm{lb}$. rate, postpaid or express prepaid. 


\section{Maule's Superior Flower Seed}

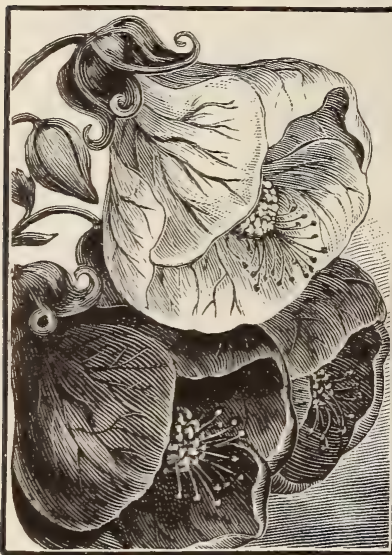

892 Abutilon, Choicest Hybrids

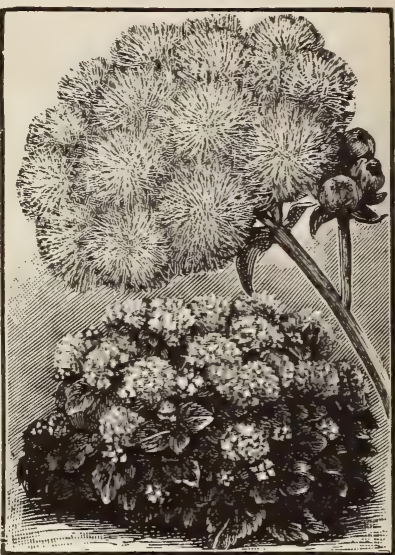

904 Ageratum, Little Blue Star

Abutilon (Flowering Maple)

Handsome decorative pot plants. 892 Choicest Hybrids (All Sorts)

$$
\begin{array}{ccc}
\multicolumn{3}{c}{\text { Postpaid }} \\
\hline 1 / 16 \text { oz. } & 1 / 8 \text { oz. } & 1 / 4 \text { oz. } \\
\$ 0.85 & \$ 1.50 & \$ 2.75
\end{array}
$$

Ageratum (Floss Flowers)

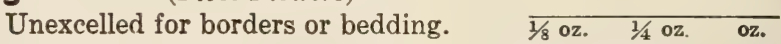
902 Dwarf Imperial, Dark blue . . . . . . \$0.15 $\$ 0.20 \quad \$ 0.50$ 903 Mexicanum, Blue................

904 Little Blue Star, Dwarf.................... $\quad .35 \quad .65 \quad 2.00$

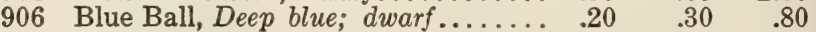

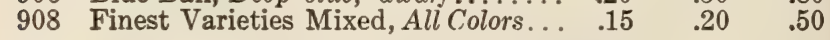

\section{Alyssum}

Popular for bedding or pots.

912 Lilac Queen, Pale lilac; dwarf......\$0.15 $\$ 0.50 \quad \$ 1.50$

914 Little Gem (Carpet of Snow), White. $\quad .15 \quad .50 \quad 1.50$

916 Saxatile compactum (Perennial), Yellow.......................

918 Sweet, The well known white sort....

Anchusa (Cape Forget-Me-Not)

Desirable for cut flowers.

928 Capensis, Deep blue, white center....

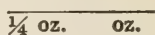

$\$ 0.15 \$ 0.40$

Arctotis (Blue-Eyed African Daisy)

Easily grown. Valuable for cutting. 966 Grandis, White petals, reverse lilac...

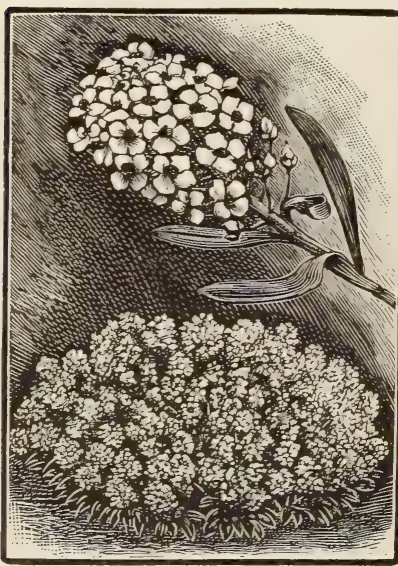

914 Alyssum, Little Gem

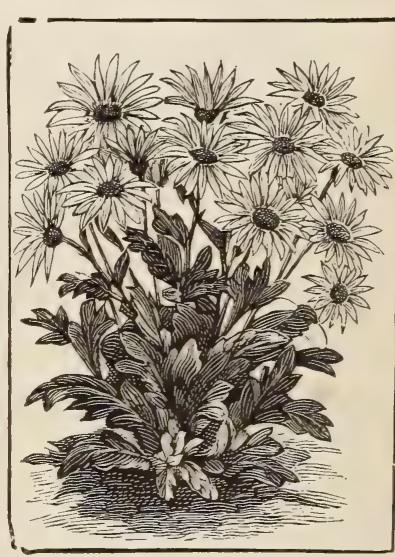

966 Arctotis, Grandis

"The Old Reliable Seed House" 


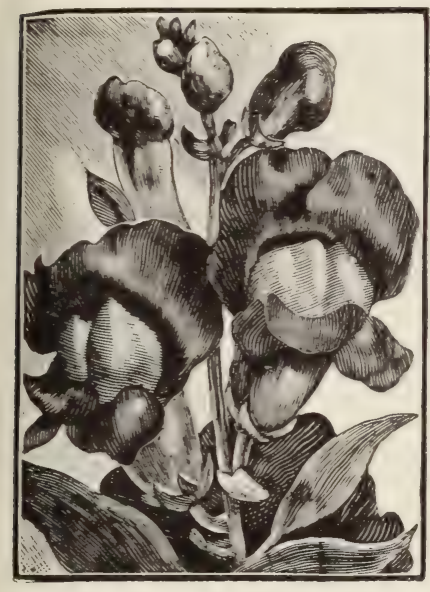

Antirrhinum, Maximum Giant

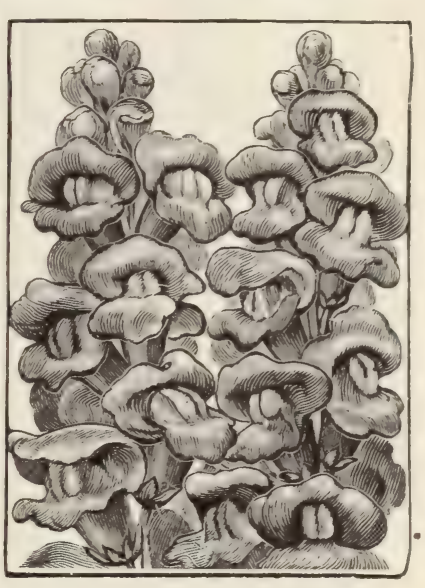

Antirthinum, Semi-Tall

Antirrhinum (Snapdragon)

Popular for bedding. Largely used

for cutting.

Maximum New Giant Flowered

\begin{tabular}{c} 
Postpaid \\
\hline $1 / 16$ oz. $1 / 8$ oz. $1 / 4$ oz. $\quad$ oz.
\end{tabular}

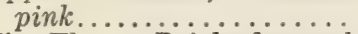

931 Fire Flame, Bright fiery red

933 The Rose, Rose-pink

934 Snowflake, Creamy white.

938 Orchid, Mauve-pink.......

943 Canary Bird, Yellow.

$\begin{array}{llll}\$ 0.20 & \$ 0.35 & \$ 0.65 & \$ 2.00\end{array}$

947 Sunnybrook, Deep golden yellow................

951 Ruby, Deep ruby-red.....

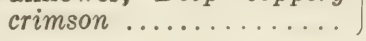

935 Mixed Colors..............

$\begin{array}{llll}.20 & .30 \quad .40 \quad 1.25\end{array}$

\section{Semi-Tall Giant Flowering}

944 Firebrand, Rich deep red..

937 Climax, Orange-yellow lip.

939 Brilliant Rose, Bright rose.

941 Purity, Large white flowers

949 Philadelphia Pink, Pure

954 Golden Mina............. Intense

Golden Monarch, Intense

1/8 oz. $1 / 4$ oz. oz.

$\$ 0.20 \quad \$ 0.35 \quad \$ 1.00$

940 Semi-Tall, Mixed Colors.

\section{Asparagus}

Extremely pretty window or house $\frac{\text { Per }}{\text { Per }}$ plants.
100 Seeds 1,000 Seeds

974 Plumosus nanus, Valuable in bouquets. $\$ 0.25 \quad \$ 1.00$

976 Sprengeri, Fine for baskets, vases, etc. $\quad \begin{array}{rr}\$ 20 & .90\end{array}$

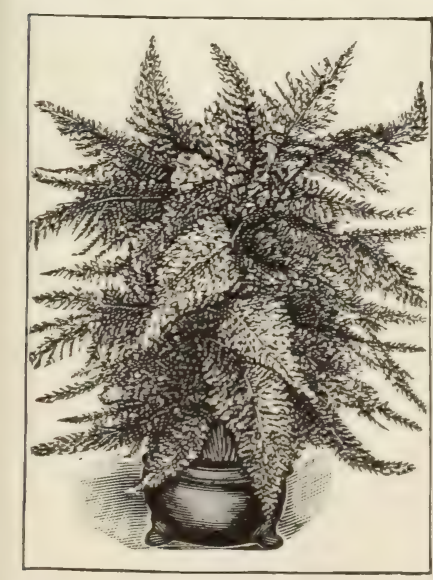

974 Asparagus, Plumosus Nanus

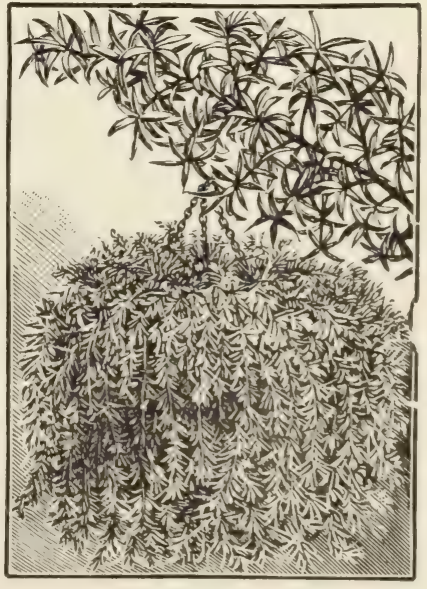

976 Asparagus, Sprengerii

All flower seeds are sent postpaid at these special prices 


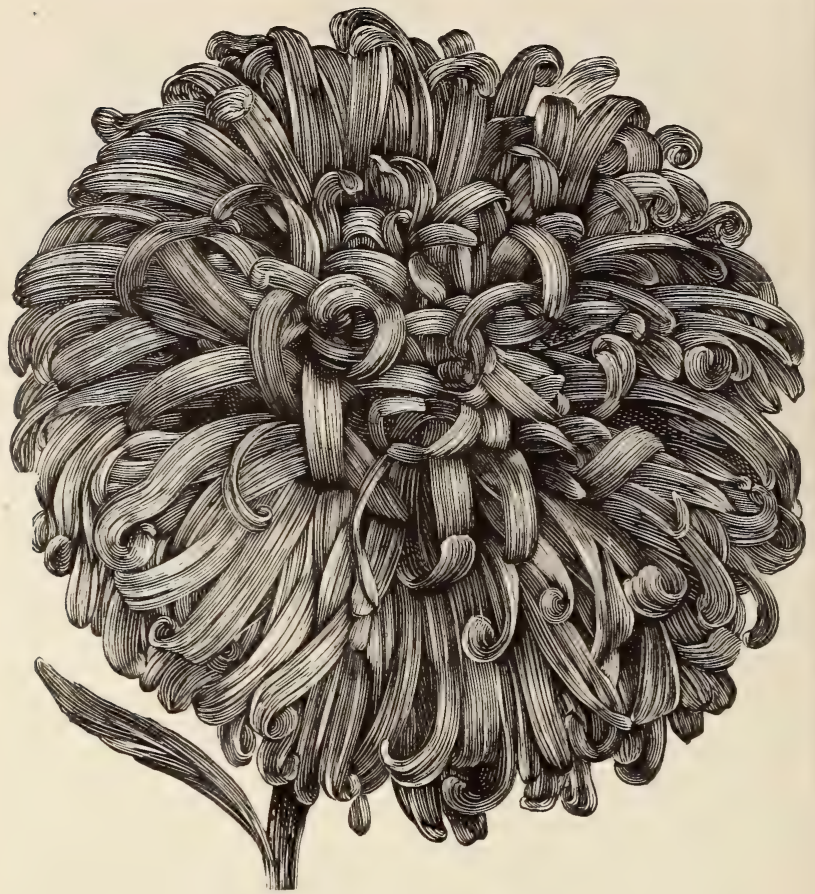

California Giant Aster

\section{"California Giants" Asters}

The flowers are of the Crego type, blooming just before the late Branching. Excellent for cut flowers.

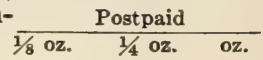

993 Light Blue................

994 Peach Blossom................

995 Dark Purple.................

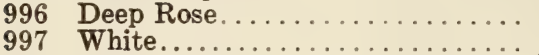

998 Mixed Colors..................... $.35 \quad .65 \quad 2.00$

\section{Giant Astermum Asters}

A beautiful strain of the Comet type. The flowers are large and have long, gracefully curled petals. Particularly appreciated as cut flowers.

\section{$\$ 0.40 \quad \$ 0.75 \quad \$ 2.25$}

Lavender................. )

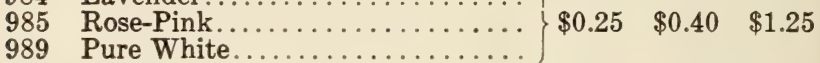

990 Mixed Colors.................

\section{Crego's Giant Comet Asters}

The flowers are as fine as Chrysanthemums, often measuring 5 inches across. $1 / 2$ oz. $1 / 4$ oz. $\quad$ oz.

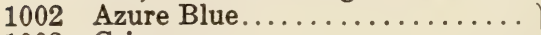

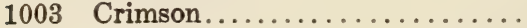

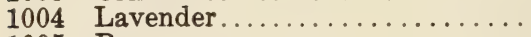

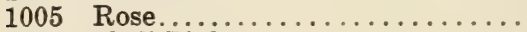

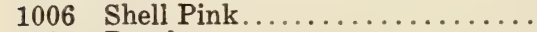

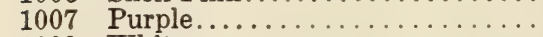

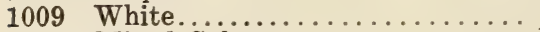

1010 Mixed Colors..................... .20 $\quad .35 \quad 1.00$

\section{Maule's Earliest Blooming Asters}

Blooms in July, two weeks earlier than most

\begin{tabular}{|c|c|c|c|c|}
\hline & the other types. & $\sqrt[1 / 8 \mathrm{oz}]{ }$ & $1 / 4$ oz. & oz. \\
\hline 1026 & Crimson.............. & & & \\
\hline 1027 & Lavender $\ldots \ldots \ldots \ldots \ldots \ldots \ldots$ & & & \\
\hline 1028 & Flesh Pink . . . . . . . . . . . . & $\$ 0.20$ & $\$ 0.35$ & $\$ 1.00$ \\
\hline $\begin{array}{l}1029 \\
1030\end{array}$ & 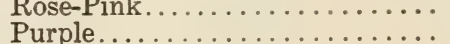 & & & \\
\hline 32 & 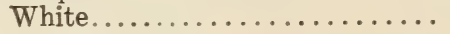 & & & \\
\hline 35 & Mixed Colors................ & .15 & .25 & .80 \\
\hline
\end{tabular}




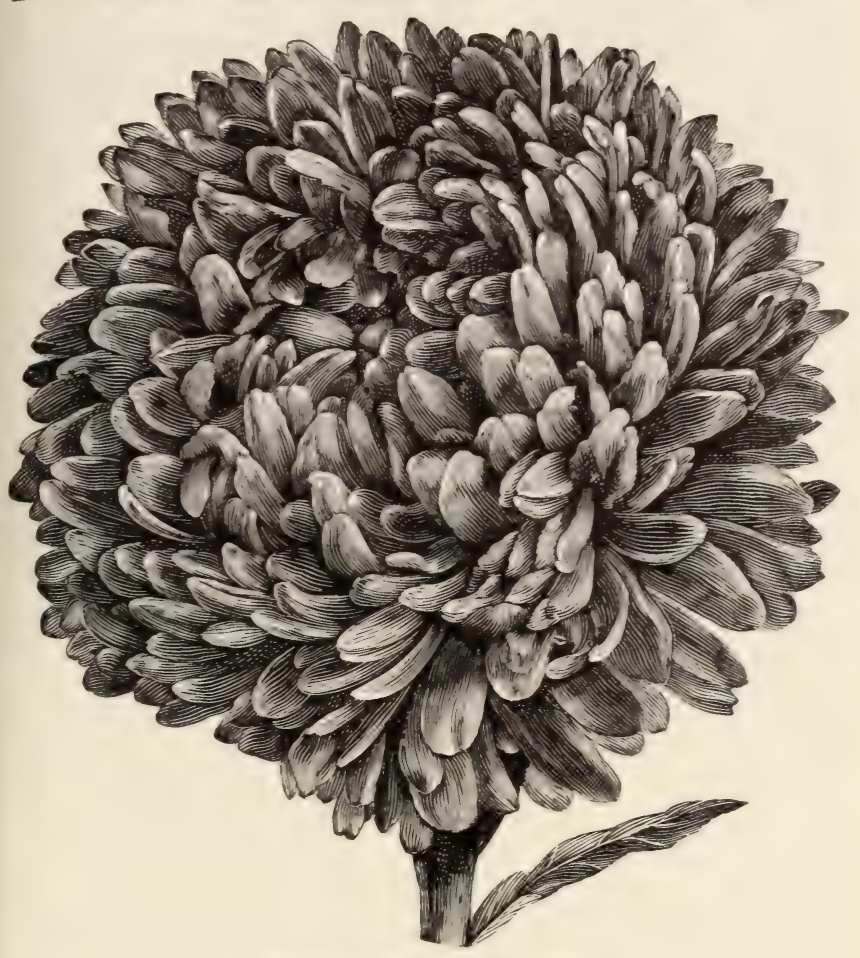

Maule's Giant Branching Aster

\section{Early American Beauty Asters}

Flowers are of the peony-shaped type on long stems. Excellent for cut flowers. Come into bloom about same time as Crego Asters.

982 American Beauty, Carmine-rose

1013 Azure Blue...................

1014 Shell Pink.

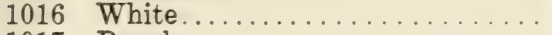

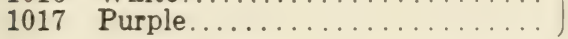

1020 Mixed Colors............... .25 $\quad .40 \quad 1.25$

\section{Maule's Giant Branching Asters}

These admirable Asters are usually at their best in September. The tendency to form numerous branches gives the class its name. The flowers are 4 inches in diameter, borne on long stems and are showy.

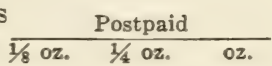

1102 Azure Blue..... . . . . . . . . . . .

1103 Crimson......................

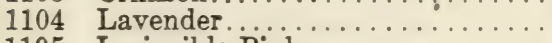

1105 Invincible Pink.............

1106 Shell Pink (Mary Semple)......

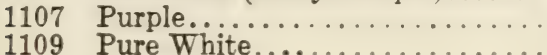

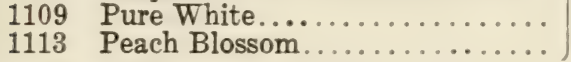

1110 All Colors, Mixed...............

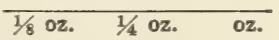

$\$ 0.20 \quad \$ 0.35 \quad \$ 1.00$

\section{Miscellaneous Asters}

All are large flowering with long stems.

Useful for bouquets or for cutting.

980 Anniversary Mixture, All types $1 / 8$ oz.

1012 Crimson Giant, Late branching type.

1080 Peerless Pink, Late branching type. .

1089 Improved Giant, California Sunshine, Mixed Colors.............

1100 Early Branching or Royal, Mixed Colors ......................

1111 Heart of France, Ruby-red........

1112 Sensation, Red; early branching type.

\begin{tabular}{rrr}
\hline $1 / 8$ oz. & \multicolumn{1}{c}{$1 /$ oz. } & \multicolumn{1}{c}{ oz. } \\
$\$ 0.25$ & $\$ 0.40$ & $\$ 1.25$ \\
.25 & .40 & 1.25 \\
.25 & .40 & 1.25 \\
.55 & 1.00 & 3.00 \\
.20 & .35 & 1.00 \\
.30 & .50 & 1.50 \\
.25 & .40 & 1.25
\end{tabular}




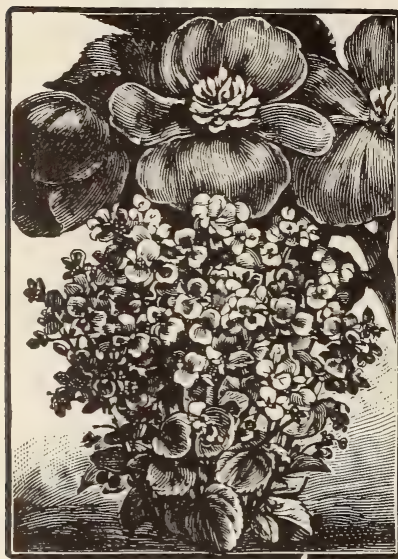

1135 Begonia, Luminosa

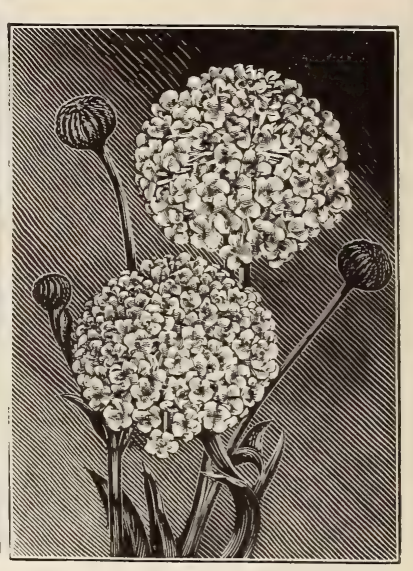

1151 Blue Lace Flower

\section{Begonia}

Desirable pot plants.

$$
\frac{\text { Postpaid }}{1 / 128 \text { oz. } 1 / 64 \text { oz. } 1 / 32 \text { oz. } 1 / 16 \text { oz. }}
$$

1134 Tuberous Rooted, Double. $1 / 128.00 \$ 3.75$

1135 Luminosa, Fiery dark

$\begin{array}{lllll}\text { scarlet.......................... } & .60 & 1.10 & \$ 1.75\end{array}$

$\begin{array}{lllll}1136 & \text { Prima Donna, Carmine-pink } & .85 & 1.50 & 2.75\end{array}$

1138 Semperflorens, Single Mixed. $\quad \begin{array}{rrrr}.35 & .60 & \$ 1.10\end{array}$

\section{Blue Lace Flower}

Handsome cut flower

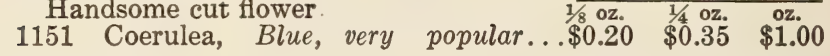

\section{Cactus}

115

\section{Calceolaria (Pouch Flower)}

Highly ornamental pot plants.

1155 Hybrida, Fine Mixed..........

\begin{tabular}{cc}
\hline 100 Seeds & 1,000 Seeds \\
$\$ 0.20$ & $\$ 1.60$
\end{tabular}

Calendula (Cape or Pot Marigold)

\section{Succeeds everywhere. Blooms con-}

tinuously.

1156 Ball's Gold, Deep golden yellow.................. \$0.30 $\$ 0.50$

\begin{tabular}{ll}
\hline $1 / 128$ oz. & $1 / 64$ oz. \\
$\$ 1.25$ & $\$ 2.00$
\end{tabular}

1157 Lemon Queen, Double lemon-

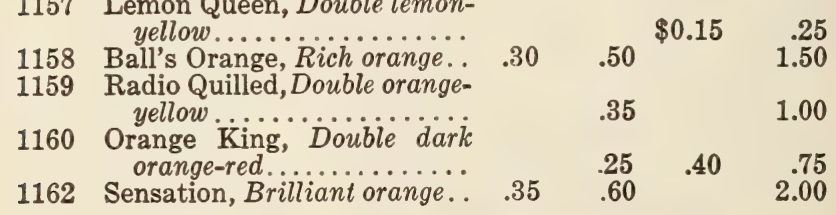

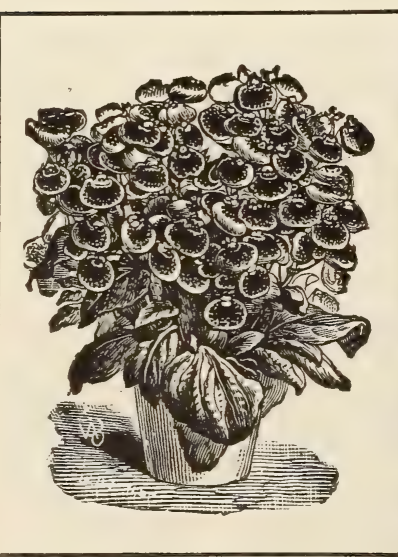

1155 Calceolaria

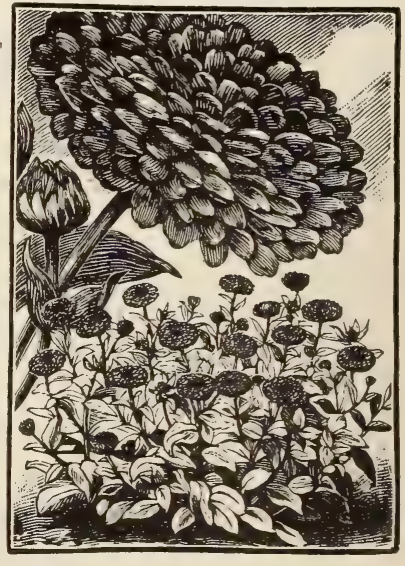

1160 Calendula, Orange King

"The Old Reliable Seed House" 


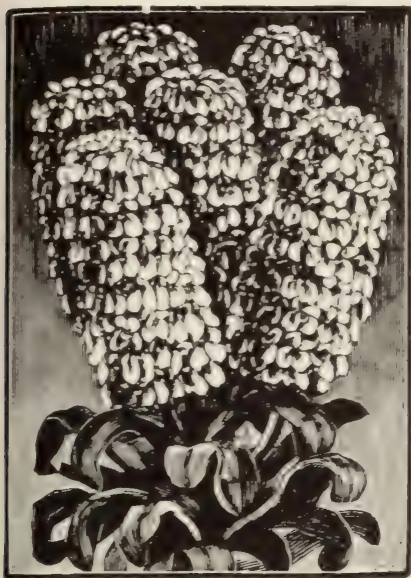

1183 Candytuft, Hyacinth Flowered

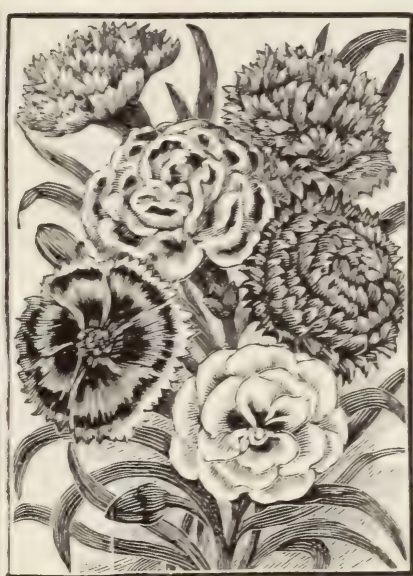

1191 Carnation Chabaud's Mixed

\section{Candytuft}

Quick growing. Valuable for cutting.

1182 Rose Cardinal, Brilliant rosy red.

1183 Hyacinth Flowered, Pure white.

1185 Mixed, All Colors. All shades. 1186 Sempervirens, White, dwarf; pe-

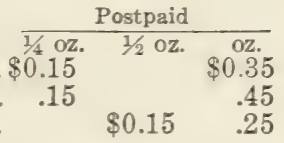

\section{Calliopsis}

Excellent cut flowers.

1165

Tall Mixed

$\begin{array}{cr}1 / 2 \text { oz. } & \text { oz. } \\ \$ 0.20 & \$ 0.35\end{array}$

1168

Dwarf Mixe

.20

.35

\section{Carnations}

For pot culture or cutting.

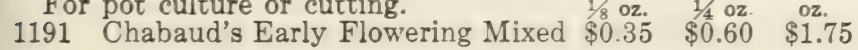

1192 Chabaud's White..............

1193 Chabaud's Rose................

1194 Chabaud's Scarlet...............

1199 Hardy Border, Double Mixed.

$.35 \quad .60 \quad 1.75$

Celosia Childsii (Chinese Woolflower)

Desirable for cutting.

1201 Crimson

$\begin{array}{lll}\$ 8.25 & \$ 0.46 & \$ 0.25\end{array}$

Plumed Celosia (Cockscomb)

1207 Thompson's Magnifica, Mixed.... $\quad .25 \quad .75$

1208 Spicata, Silver white, carmine tips..

1209 Pride of Castle Gould, Mixed Colors $\quad .45 \quad .80 \quad 2.25$

\section{Crested Celosia Dwarf Habit}

1205 Empress, Purple-red flowers........ . .30 $\quad .50 \quad 1.50$

1204 Cristata Mixed, All best colors....... $\quad .20 \quad .30 \quad .80$

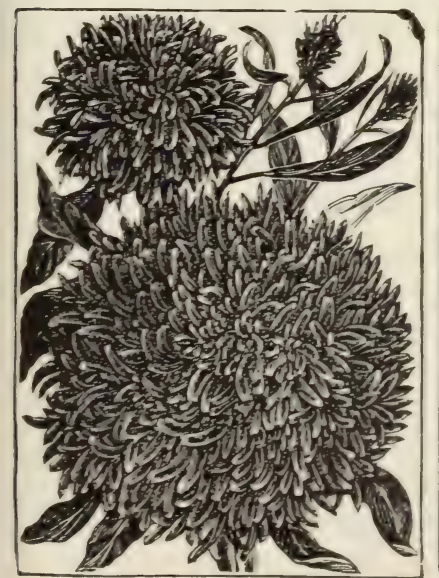

Celosia, Chinese Woolflower

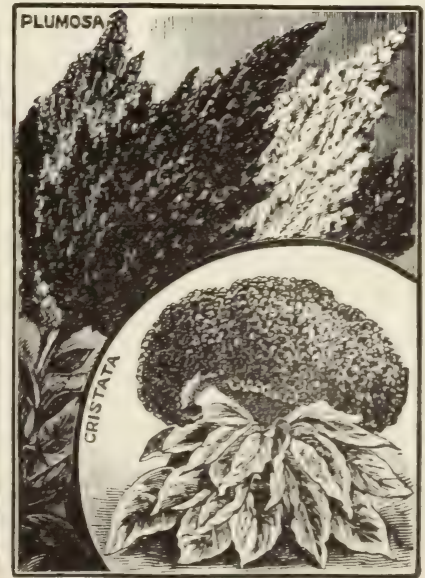

Celosia

All flower seeds are sent postpaid at these special prices 


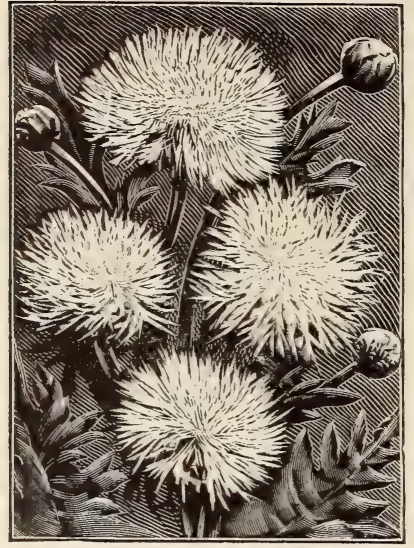

1211 Centaurea, Imperialis

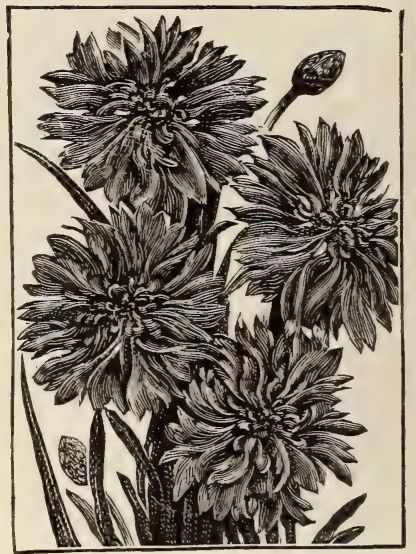

1213 Centaurea, Cyanus, Double Blue

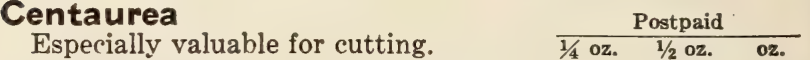

1206 Montana..................\$0.20 $\$ 0.35 \quad \$ 0.60$

1211 Imperialis Mixed, All colors........ $\quad .15 \quad .25 \quad .45$

1215 Gymnocarpa (DustyMiller)........ $\quad .20 \quad .50$

Centaurea cyanus (Corn Flower)

Well known to every grower for cutting.

1213 Double Blue, Extra large......... . .15 $\quad .40$

1218 Double Mixed, All colors............... $.15 \quad .30$

\section{Chinese Lantern}

Makes fine winter decorations.

1220 Physalis Franchetti.

1/40z. oz.

$\$ 0.25 \$ 0.75$

Chrysanthemum (Painted Daisics)

Extremely showy, Daisy-like flowers.

1223 Coronarium, Finest double mixed .........\$0.15 \$0.25

1226 Nivellii, Yellow Daisy-like flowers......... .35 1.00

1227 Single Finest Mixed, Many colors......... . . .15 $\quad .30$

\section{Cineraria (Cape Aster)}

A handsome, free flowering pot plant.

1234 Tall Finest Mixed, Large flowering. $\$ 0.60 \quad \$ 1.00 \quad \$ 1.75$

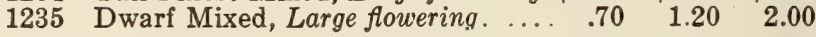

\section{Coleus}

Beautiful colored leaves. For pot culture.

1/32 oz. $1 / 16$ oz. $1 / 8 \mathrm{oz}$

1244 Mammoth Rainbow Mixture.....\$0.85 $\$ 1.50 \quad \$ 2.50$

1246 Choicest Hybrids, Small leaves..... $\quad .50 \quad \begin{array}{rr}\$ 1.50 & 1.50\end{array}$

1247 Finest Mixed, Tall growing........ $\quad .50 \quad .80 \quad 1.50$

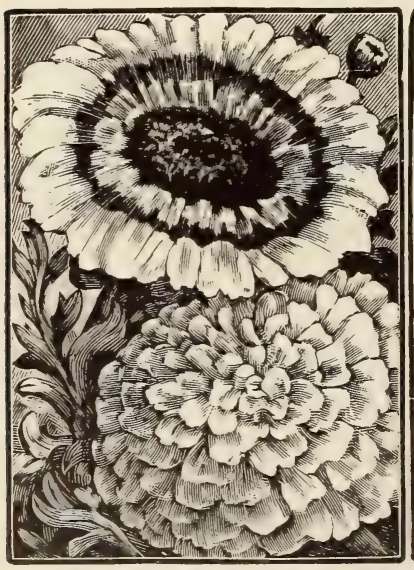

1227 Chrysanthemum, Single Mixed 1223 Double Mixed

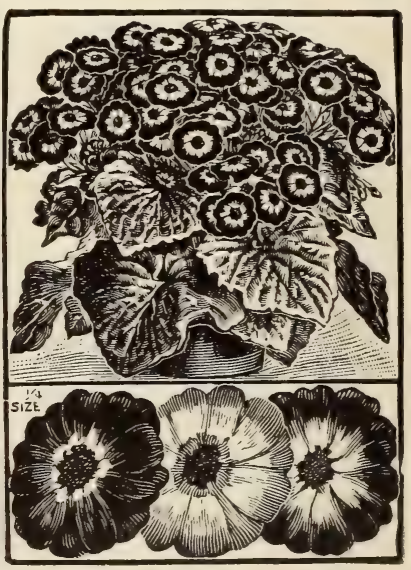

1235 Cineraria, Dwarf, Mixed 


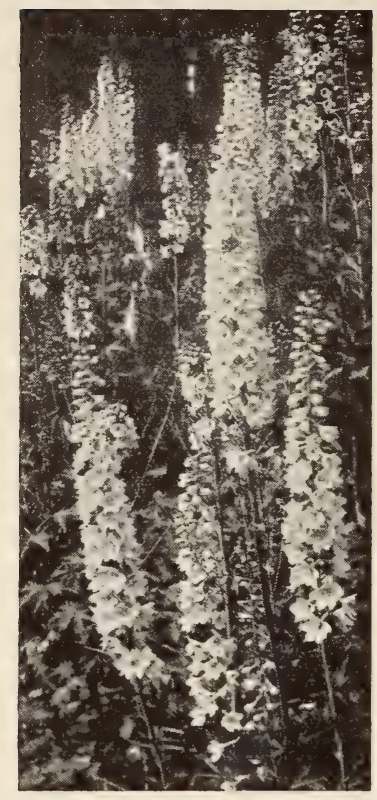

1289 Delphinium Prize Exhibition

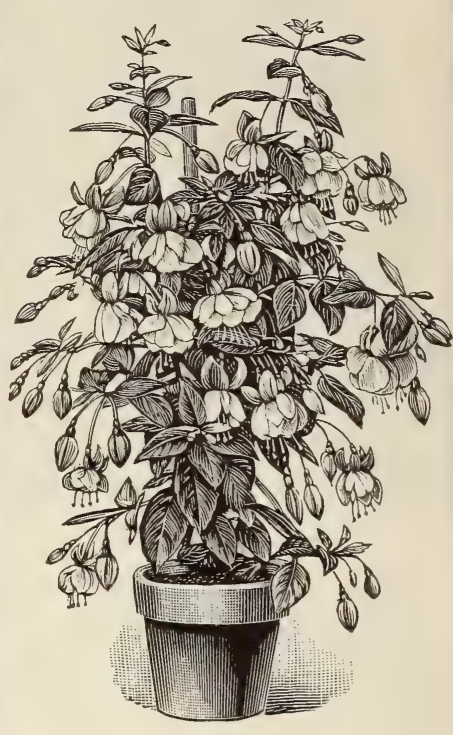
Mixture

1337 Fuchsia, Choice Mixed Hybrids

Delphinium (Hardy Larkspur)

Very popular as cut flowers. \begin{tabular}{cccc}
\multicolumn{4}{c}{ Postpaid } \\
\hline $1 / 16$ oz. & $1 / 8$ oz. $1 / 4$ oz. & oz.
\end{tabular}

1289 Prize Exhibition Mixture...\$0.60 $\$ 1.00 \quad \$ 1.75 \quad \$ 5.00$

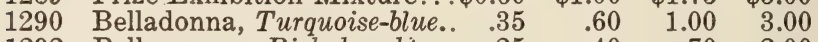

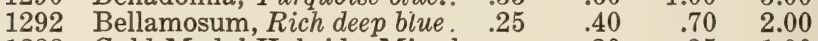

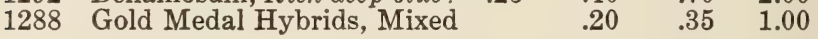

$\left.\begin{array}{l}1297 \\ 1298 \\ \text { Deep Blue............... }\end{array}\right\} \quad .50 \quad .85 \quad 1.50 \quad 4.50$

1299 Light Blue..............

$\begin{array}{llllll}1300 & \text { Wrexham Strain.......... } & .60 & 1.00 & 1.75 & 5.00\end{array}$

1301 Choice Mixed.......... $\quad .20 \quad .35 \quad 1.00$

\section{Dianthus, Hardy}

Excellent, hardy perennial pot plants. $\quad \overline{1 / 4 \mathrm{oz} .} \quad$ oz. 1308 Double Mixed, Beautiful colors.........\$ \$0.20 $\$ 0.50$

1309 Single Mixed, Splendid flowers.......... . $\quad .15 \quad .40$

\section{Dianthus, Annual (Pinks)}

Excellent for cut flowers. $\quad 1 / 4.0 z . \quad$ oz. 1294 Heddewigii, Double flowers, all colors.....\$0.20 $\$ 0.60$ 1307 Lucifer................................. $\quad .70$ 1303 Allwoodii.......... oz. $\$ 1.35$; $1 / 8$ oz. $\$ 4.25$ 1310 Plumarius, Double and Single, Mixed..... .30 $\quad .85$

Euphorbia (Snow-on-the-Mountain)

A showy cut flower or border foliage plant.

1329 Variegata, Foliage margined with white....\$0.15 $\quad \begin{array}{cc}\frac{1 / 40.40}{\mathrm{oz}_{0}} & \$ 0.40\end{array}$ 1328 Heterophylla, Fire-on-the-Mountain...... $.20 \quad .60$

\section{Fuchsia}

Desirable pot plants. $\overline{50 \text { Seeds } 100 \text { Seeds }}$ 1337 Choice Mixed Hybrids............ \$0.50 \$0.75

Gaillardia (Blanket Flower)

Largely used for cutting. $\begin{array}{lll}1 / 2 \text { oz. } & 1 / 2 \text { oz. } & \text { oz. }\end{array}$ 1338 Lorenziana, Double Mixed, Annual.\$0.15 $\$ 0.25 \quad \$ 0.40$ 1341 Fine Mixed Perennial, All kinds... $\quad .15 \quad .25 \quad .40$

\section{Geranium}

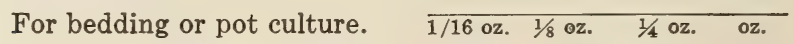
1348 Zonale Mixed, Many colors. $\$ 0.25 \quad \$ 0.40 \quad \$ 0.70 \quad \$ 2.25$ 


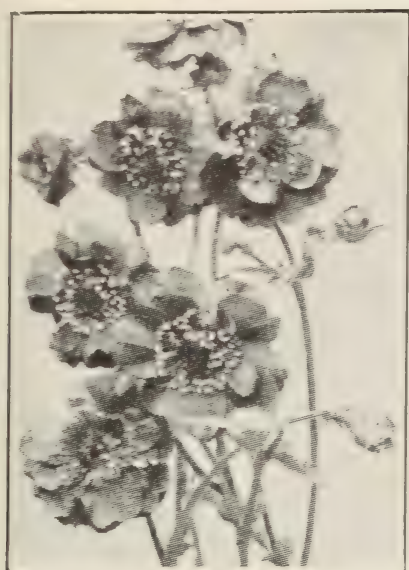

1351 Geum, Mrs. Bradshav

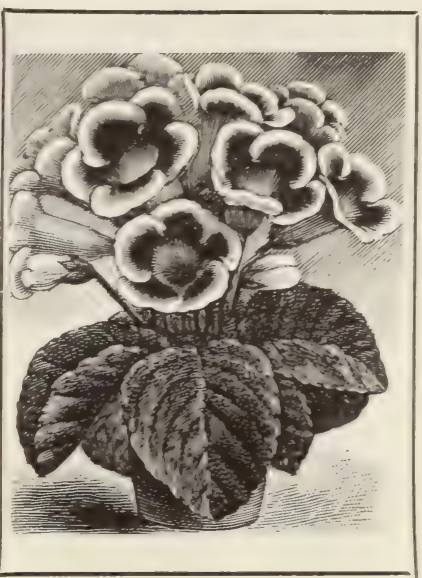

1358 Gloxinia, Hybrids, Mixed

\section{Geum}

Pretty cut flowers.

$$
\begin{array}{lrc}
\multicolumn{3}{c}{\text { Postpaid }} \\
\hline 1 / 16 \text { oz. } & 1 / 4 \text { oz. } & \text { oz. } \\
\$ 0.20 & \$ 0.50 & \$ 1.50
\end{array}
$$

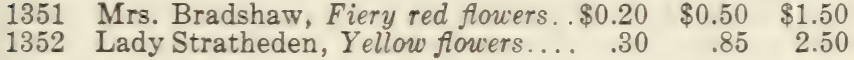

\section{Globe Amaranth}

Clover-like flowers of the everlasting type.

1356 Fine Mixed, White, purple, striped, etc....\$0.15 $\$ 0.30$

\section{Gloxinia}

Excellent decorative pot plants.

1358 Choicest Hybrids, Mixed colors.

\begin{tabular}{cr}
\hline $1 / 128$ oz. & $1 / 64$ oz. \\
$\$ 0.75$ & $\$ 1.25$
\end{tabular}

\section{Gypsophila (Baby's Breath)}

Valuable for use in bouquets.

1368 Giant, White; annual..........\$0.15 $\$ 0.45 \quad \$ 1.50$

1369 Carmine, Annual.............. $.20 \quad .60 \quad 2.00$

1370 Rose, Annual.............................. $\quad .45 \quad 150$

1371 Paniculata, Misty white pan-

$$
\text { icles; perennial............ }
$$

1383 Paniculata fl. pl., Double

white; perennial........\$0.35 $\$ 0.65 \quad 1.20 \quad-$

\section{Helichrysum (Straw-Flover)}

Highly prized for winter bouquets. $\overline{1 / 40 z . \quad 0 z . ~}$ 1374 Tall Double Mixed, All colors..........\$0.20 \$0.50

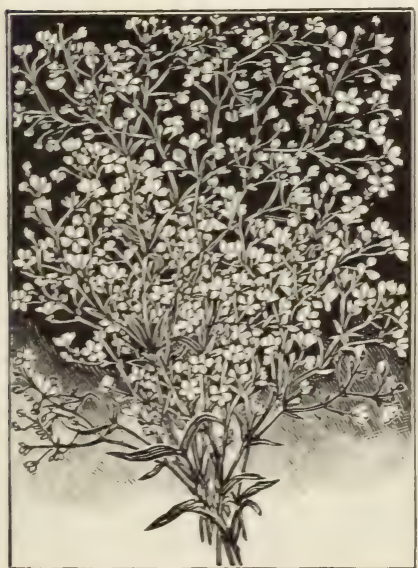

1371 Gypsophila, Paniculata

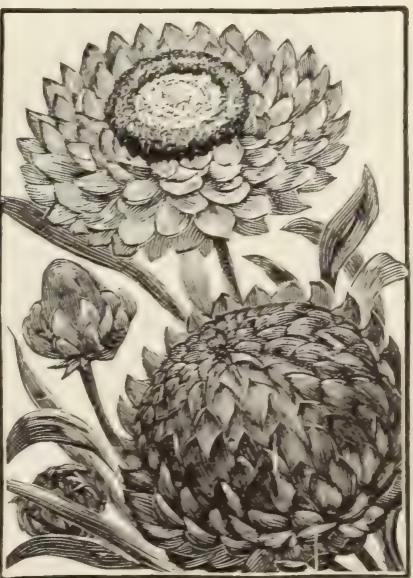

1374 Helichrysum, Double Mixed 

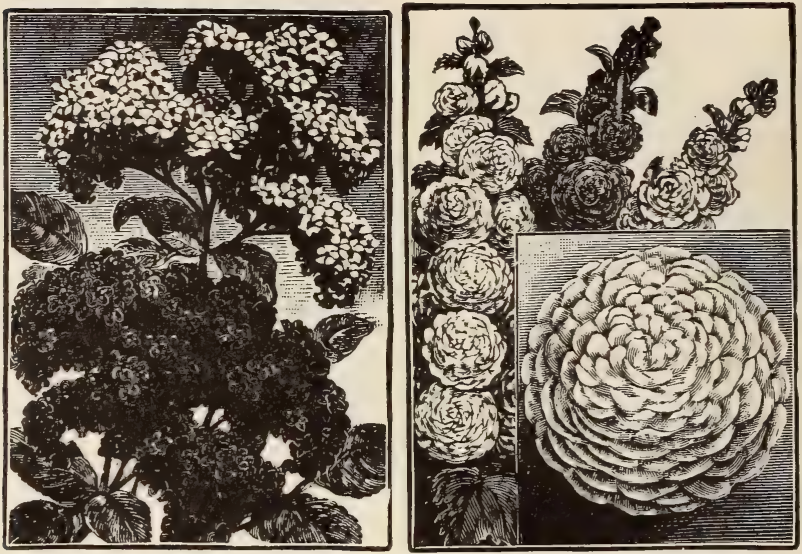

1375 Heliotrope, Lemoine's Giant
Hybrids

1990 Hollyhock, Double Fine Mixed

Heliotrope (Cherry Pie)

A useful cut flower or pot plant.

1375 Lemoine's Giant Hybrids.

\begin{tabular}{ccc}
\multicolumn{4}{c}{ Postpaid } \\
\hline $1 / 8$ oz. $1 / 4$ oz. oz.
\end{tabular}

1380 Choice Mixed, Many varieties...... $.30 \quad .30 \quad \begin{array}{rr}\$ 0 & .50\end{array}$

\section{Hollyhock}

Popular old-fashioned plants.

1392 Newport Pink, Best double pink.

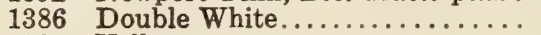

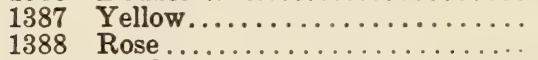

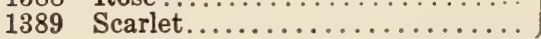

1390 Double Fine Mixed, All colcrs.... .20

Impatiens (Sultan's Balsam)

A continuous blooming pot plant. $\overline{1 / 32 \mathrm{oz} .1 / 16 \mathrm{oz} .1 / 30 z}$ 1401 Large Flowering Hybrids, Mixed..\$0.70 $\$ 1.25 \$ 2.25$

Japanese Iris (Flowering Flag)

A useful bedding plant.

1411 Mixed Colors...................\$0.25 \$0.60

\section{Jerusalem Cherry}

Attractive pot plant.

1413 Solanum capsicastrum nanum. ...\$0.20 $\$ \$ 0.35 \quad \$ 1.00$

\section{Order by Number or Name}

Order all seeds by correct number or by name, but if both are given mistakes are less liable to occur.

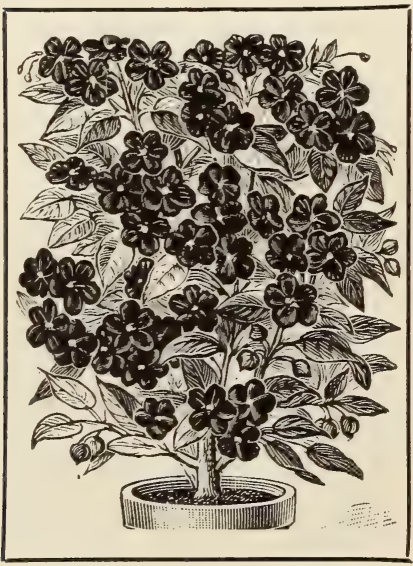

1401 Impatiens, Large Flowering Hybrids, Mixed

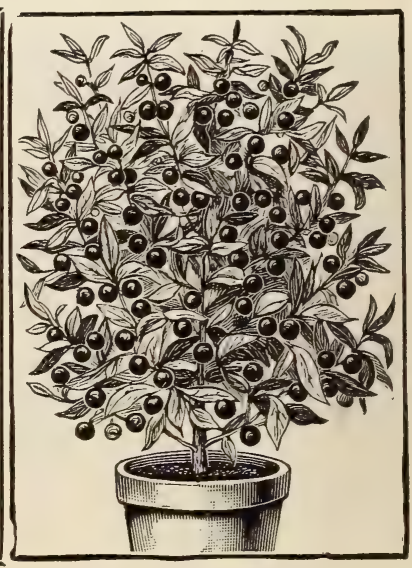

1418 Jerusalem Cherry 


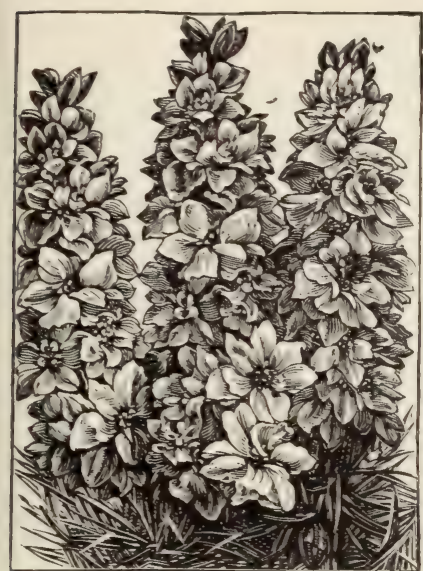

1420 Larkspur, Exquisite

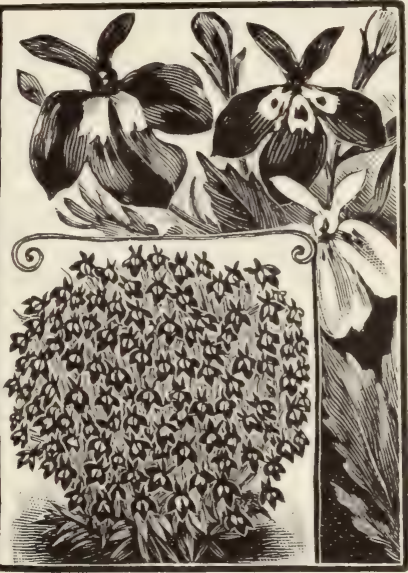

1432 Lobelia, Mixed Varieties

Larkspur (Annual Varieties)

Bloom profusely. Last well when cut. $\frac{\text { Postpaid }}{1 / 4 \text { oz. Oz. }}$ 1419 Dark Blue .......................\$0.20 \$0.50

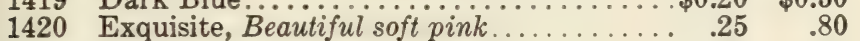

1421 Carmine.............................. $.20 \quad .50$

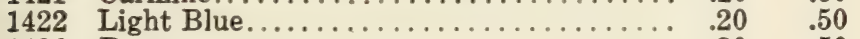

1426 Rose ........................... $.20 \quad .50$

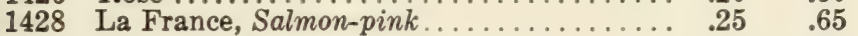

1435 White Spire....................... $25 \quad .80$

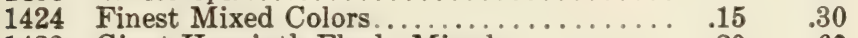

1423 Giant Hyacinth Flwd., Mixed............ $\quad .20 \quad .60$

For Perennial Larkspur, see Delphinium.

Lavandula (True Lavender)

When dried, used as perfume.

1425 Vera, Fragrant blue flowers ........... \$0.25 $\$ 0.60$

\section{Lobelia}

Desirable for bedding or pots.

1430 Compacta Crystal Palace, Deep blue. $\$ 0.35 \quad \$ 0.60 \quad \$ 1.75$

1432 Mixed Varieties................ $\quad .20 \quad .30 \quad .80$

\section{Lunaria (Honesty)}

Grown for its silvery white seed pods. $\overline{1 / 4 \mathrm{oz} . \quad \mathrm{oz} .}$ 1436 Biennis, Pinkish-purple flowers .........\$0.20 \$0.60

\section{Lupinus}

Excellent for cut flowers.

1444 Annual Mixed...................

1443 New Giants, Mixed............ $\$ 0.85$

1445 Perennial Mixed................ .15

$\begin{array}{rr}1 / 2 \text { oz. } & \text { oz. } \\ \$ 0.20 & \$ 0.30 \\ & 2.50\end{array}$

2.50

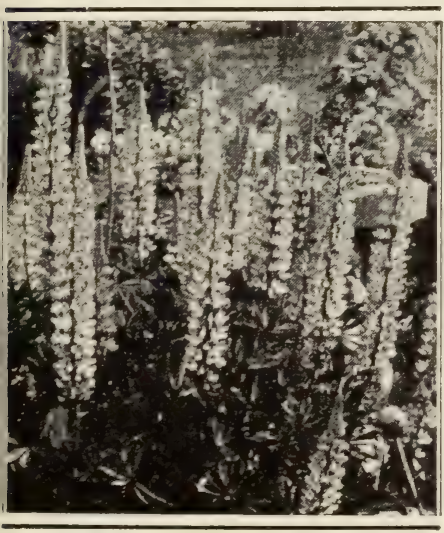

Lupinus, Mixed

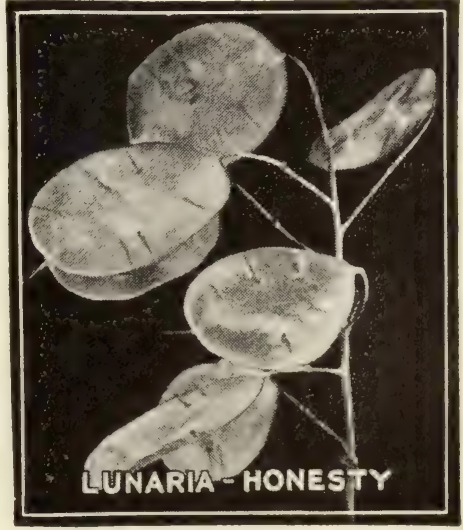

Lunaria-Honesty 


\section{Order by Number or Name}

Order all seeds by correct number or by name, but if both are given mistakes are less liable to occur.

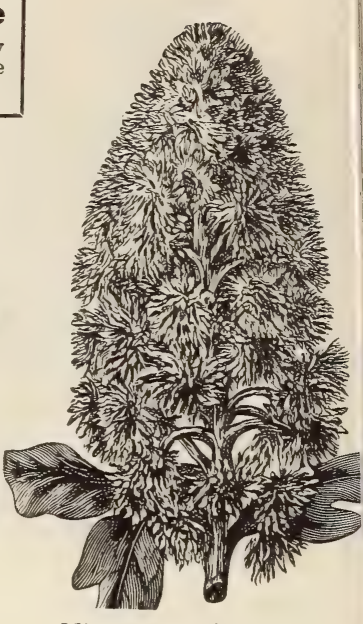

1455 Guinea Gold Marigold

1465 Mignonette, Red Goliath

\section{Marigold, French Dwarf}

One foot high; fine for pots or borders. $\frac{\text { Postpaid }}{1 / 40 z . \quad \text { oz. }}$

1450 Golden Ball, Golden yellow............\$0.20 \$0.50

1451 Legion of Honor or Little Brownie. . . . . . .15 $\quad .40$

1452 Double Mixed................... .15 $\quad .40$

1448 Pride of the Garden, Double yellow ..........

\section{Double Tall African Marigold}

Two and one-half feet high. Desirable cut flowers.

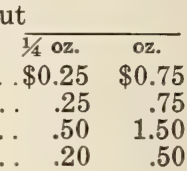

1453 Lemon Ball Tall, Double lemon colored.... \$0.25 \$0.75

1454 Orange Ball Tall, Rich golden orange..... $.25 \quad .75$

1455 Guinea Gold, Orange and gold.......... $\quad .50 \quad 1.50$

\section{Mignonette}

The best and most useful sorts for florists' use. $\overline{1 / 4 \mathbf{o z} . \quad \mathrm{oz}}$. 1465 Red Goliath, Giant spikes, extra fine......\$0.25 \$0.75

1474 Finest Mixed, All varieties............. $.15 \quad .40$

\section{Mimulus}

Curious house plants. $\quad \overline{1 / 8 \mathrm{oz} .} 1 \frac{1 / 4 \mathrm{oz}}{}$ 1478 Tigrinus grandiflorus, Monkey Flower .... \$0.30 $\$ 0.50$

\section{Myosotis (Forget-Me-Not)}

Excellent for pots or borders. Early flowering.

1490 Semperfiorens ............... \$0.30 $\$ 0.50 \quad \$ 1.50$

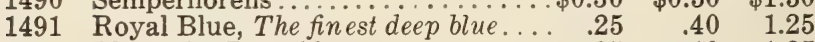

1492 Alpestris, Dwarf blue .................25 $\quad .40 \quad 1.25$

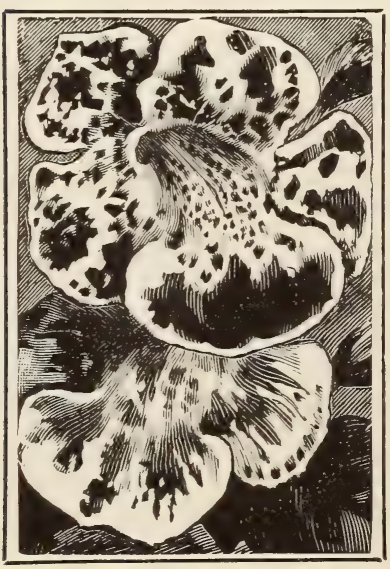

1478 Mimulus, Tigridus

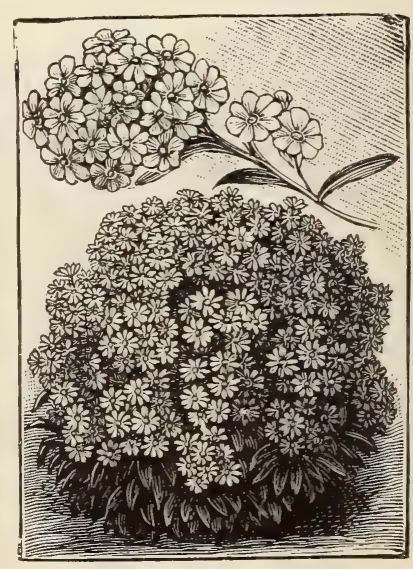

1491 Myosotis, Royal Blue

"The Old Reliable Seed House" 


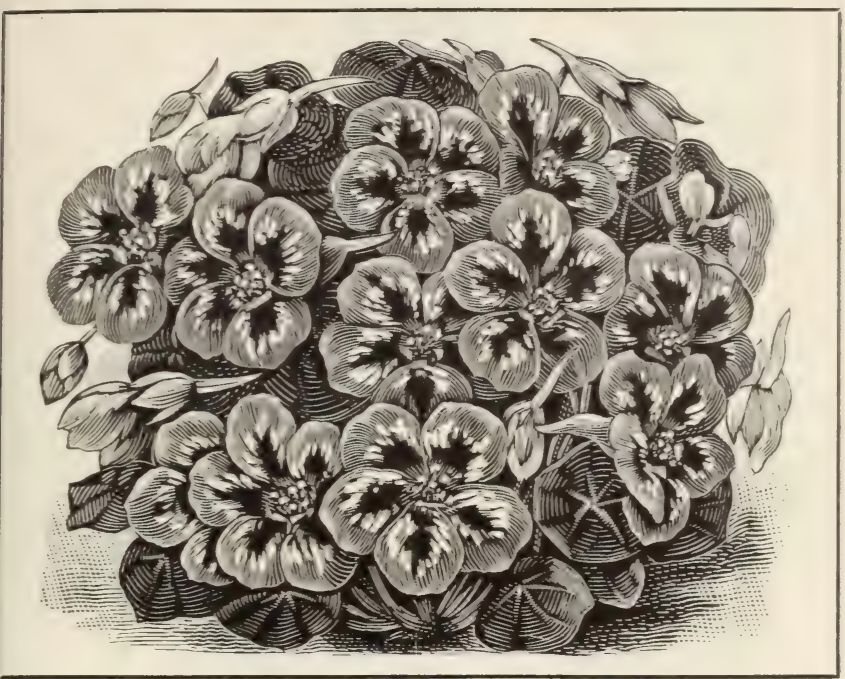

Nasturtium, Dwarf or Tom Thumb

\section{Nasturtium, Dwarf or Tom Thumb \\ For pots, borders, or rockeries. \\ Postpaid \\ 1558 Finest Mixed, A very fine mixture. $\$ 0.15$ \\ 1565 Maule's Champion Dwarf Mixture. $.20 \quad .60 \quad 1.75$

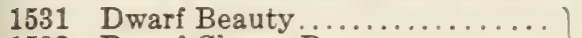 \\ 1532 Dwarf Cherry Rose........... $\quad .15 \quad .35 \quad 1.00$ \\ 1533 Dwarf King Theodore..........}

\section{Nasturtium, Tall Climbing}

Among our most popular plants.

1527 Tall Midnight..............

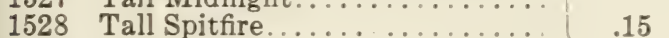

1529 Tall Sunlight..............

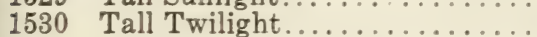

1535 Tall Choice, Mixed, Many colors. . $\quad \begin{array}{llll}.10 & .25 & .70\end{array}$

\begin{tabular}{|c|c|c|c|c|c|}
\hline \multicolumn{2}{|c|}{ Maule's Giant Pansies } & $1 / 16 \mathrm{oz}$. & $1 / 8 \mathbf{~ o z}$ & $1 / 4$ oz. & oz. \\
\hline 1594 & Oregon Giants........ & $\$ 0.90$ & $\$ 1.75$ & $\$ 3.25$ & $\$ 10.00$ \\
\hline & Giant Ruffled Masterpiece. & .30 & .50 & .80 & 2.25 \\
\hline & Giant Madame Perret..... . & & .23 & .40 & 1.25 \\
\hline & Swiss Giants, Mixed..... & .85 & 1.35 & 2.50 & 8.00 \\
\hline & Giant King of the Blacks. & & .25 & .40 & 1.25 \\
\hline 1623 & len Yellow.... & & .25 & .40 & 1.25 \\
\hline 1631 & Giant Sno & & .25 & .40 & 1.25 \\
\hline & Maule's Giant Phenomenal. & .35 & .60 & 1.00 & 3.00 \\
\hline & Giant Trimardeau, Mixed & & .25 & .40 & 1.25 \\
\hline & $\begin{array}{l}\text { Maule's Prize Mixed Giants. } \\
\text { Extra Fine Mixed Bedding }\end{array}$ & .75 & 1.35 & 2.50 & 4.00 \\
\hline & & & .20 & .35 & 1.00 \\
\hline
\end{tabular}

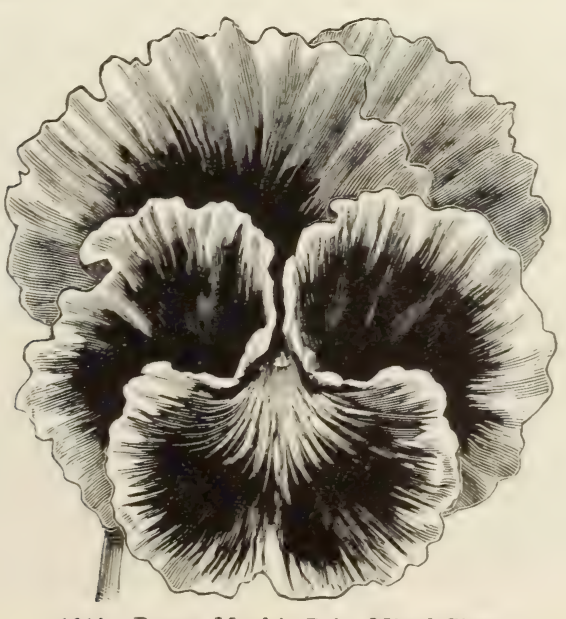

1614 Pansy, Maule's Prize Mixed Giant

All flower seeds are sent postpaid at these special prices 


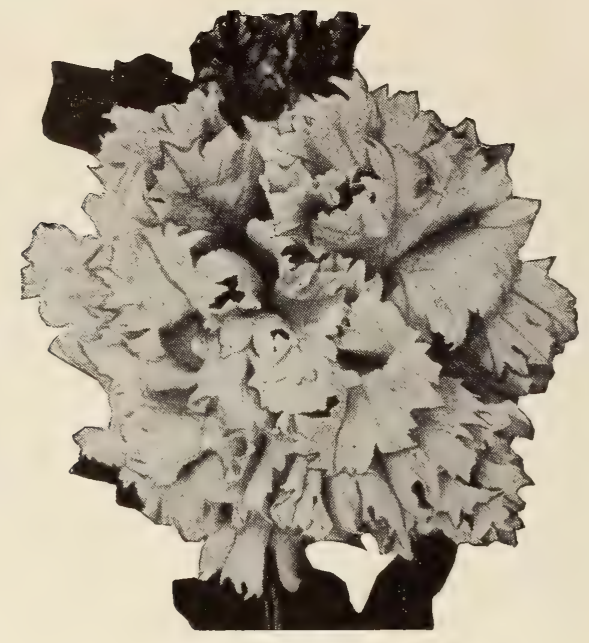

1636 Peturia, Large Double Fringed Mixed

\section{Petunia, Dwarf Bedding}

Erect growing. Fine for beds, etc. Postpaid

1632 Elk's Purple

1/128 oz. $1 / 32$ oz. $1 / 16$ oz. $1 / 40 z$.

1635 Rosy Morn, Carmine pink

1637 Rose of Heaven, Deep rose

1642 General Dodd's, Dark red

1647 Howard's Star, Crimson, white star..............

1663 Burpee's Blue, Uitramarine blue........(1/32 oz. 35c.)

1640 Dwarf Bedding, Mixed colors

1646 Belle Etoile, Striped and

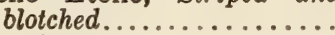

1648 Best Hybrids, Mixed........ $\$ 0.40 \$ 1.25 \quad \$ 2.25 \$ 7.00$

\section{Balcony Petunias}

Semi-trailing. For all uses. \begin{tabular}{lll}
\hline $1 / 32$ oz. $1 / 16$ oz. $1 / 4$ oz. & oz.
\end{tabular}

1639 Balcony, Star of California. $\$ 0.30 \quad \$ 0.50 \quad \$ 1.50 \quad \$ 4.50$

1641 Balcony, Blue.......... )

1643 Balcony, Crimson.........

1644 Balcony, Rose...............

1660 Balcony, Mixed Colors....

$\begin{array}{llll}.25 & .40 & 1.20 & 3.50\end{array}$

$\begin{array}{llll}.25 & .40 & 1.20 & 3.50\end{array}$

\section{Miscellaneous Petunias}

Elegant for pots.

\begin{tabular}{lll}
\hline $1 / 16$ oz. & $1 / 4$ oz. & oz. \\
$\$ 0.20$ & $\$ 0.50$ & $\$ 1.50$
\end{tabular}

1636 . $1 / 128$ oz. $1 / 32$ oz. $1 / 16$ oz.

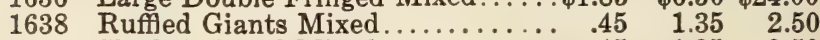

1645 Single Fringed Mixed ............ .45 $\quad 1.35 \quad 2.50$

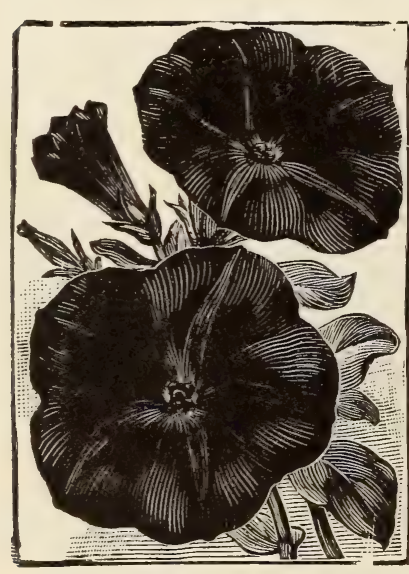

1641 Petunia, Balcony Blue

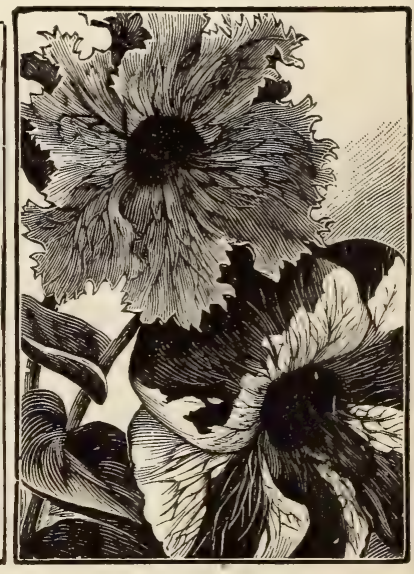

1646 Petunia, Belle Etoile

"The Old Reliable Seed House" 


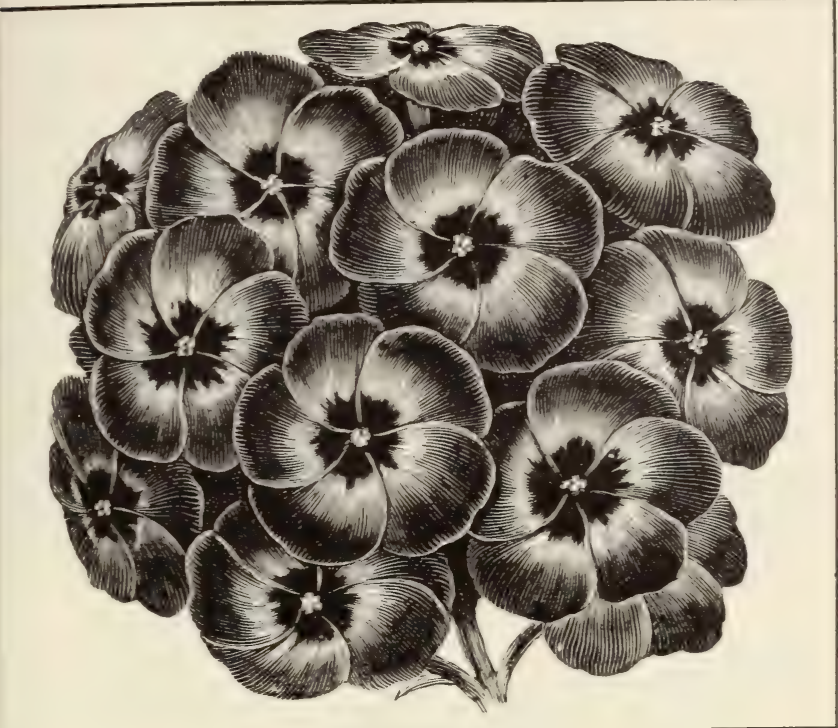

Phlox Drummundi

\section{Phlox Drummundii, Annual}

Fine for cutting. Excellent for pots or beds.

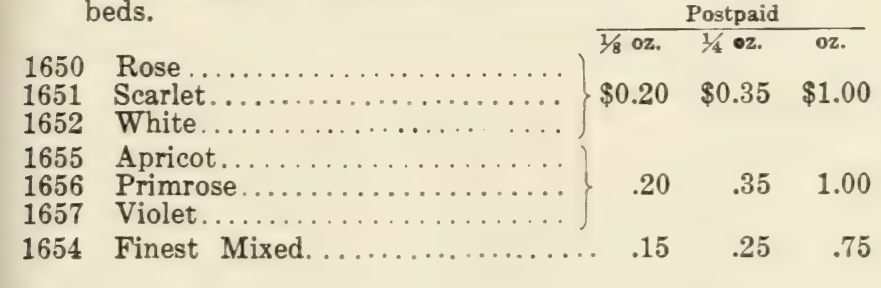

\section{Primula (Primrose)}

A popular pot plant.

1699 Obconica, Finest Mixed . . $\$ 0.40 \quad \$ 0.70 \quad \$ 1.25 \quad \$ 2.25$ $\begin{array}{llllll}1709 & \text { Chinensis, Finest Mixed... } & .50 & .85 & 1.50 & 2.50\end{array}$

Purple Feather Grass (Pennisetum)

Excellent for pots or beds.

1710 Puppelii.......................... \$0.25 $\$ 0.75$

Pyrethrum (Persian Daisy)

Beautiful cut flowers.

1711 Roseum, Single Mixed

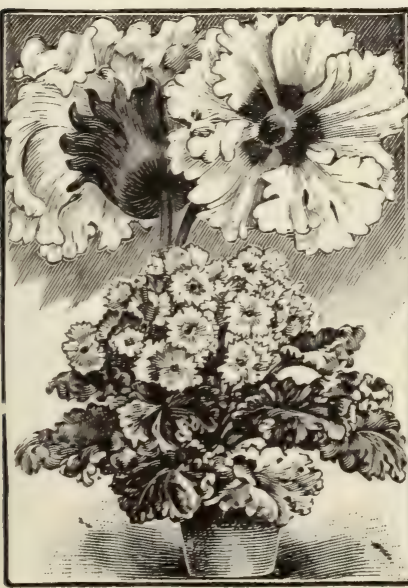

1709 Primula Chinensis, Finest Mixed

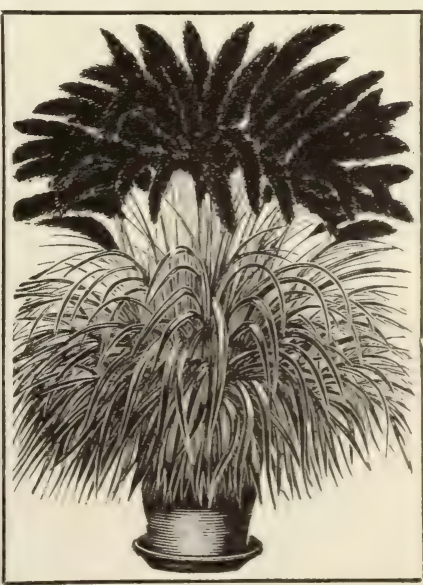

1710 Purple Feather Grass

All flower seeds are sent postpaid at these special prices 


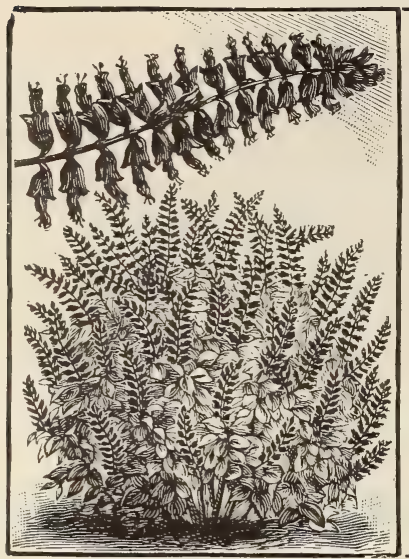

1724 Salvia America

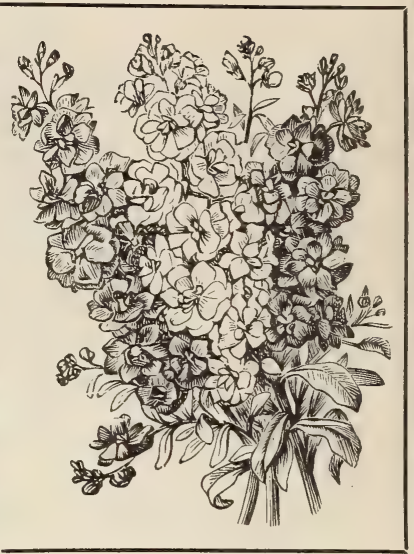

1752 Stocks, Finest Mixed

Salpiglossis (Velvet Flower)

Attractive cut flowers.

1721 Emperor, Mixed.......

$$
\begin{array}{ccc}
\multicolumn{3}{c}{\text { Postpaid }} \\
\hline 1 / 8 \text { oz. } & 1 / 4 \text { oz. } & \text { oz. } \\
\$ 0.15 & \$ 0.25 & \$ 0.75
\end{array}
$$

\section{Salvia (Scarlet Sage)}

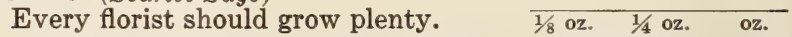
1722 Drooping Spikes, Extra choice....\$0.60 $\$ 1.10 \quad \$ 3.50$ 1723 Dwarf Bonfire, Extensively grown.. $\quad .40 \quad .75 \quad 2.25$

1724 America or Globe of Fire, Very dwarf ................. $60 \quad 1.10 \quad 3.50$

\section{Scabiosa}

Desirable for cut flowers.

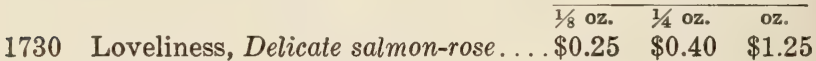
1734 Rose, Dark rose flowers...........

1737 Peach Blossom, Large pink flowers $\quad \begin{array}{lll}.50 & .50\end{array}$

1739 Red, Rich double flowers.........

1742 Large Flowering Mixed..........

\section{Shasta Daisy}

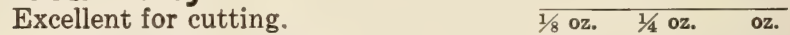
1283 Maximum, Large white daisies... . . \$0.40 $\$ \$ 0.70 \quad \$ 2.00$

\section{Statice (Sea Lavender)}

Easily dried for winter bouquets.

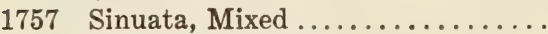

Stocks (Cut and Come Again)

Large flowering, 10 weeks.

1752 Finest Mixed, Soft and distinct $\overline{1 / 8}$ oz. $1 / 4$ oz. oz. shades .................... \$0.35 $\$ 0.60 \quad \$ 1.75$

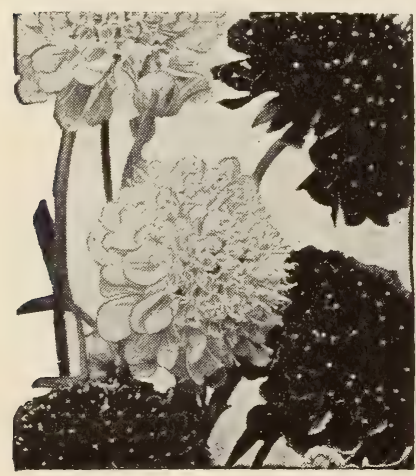

1742 Scabiosa, Mixed Double

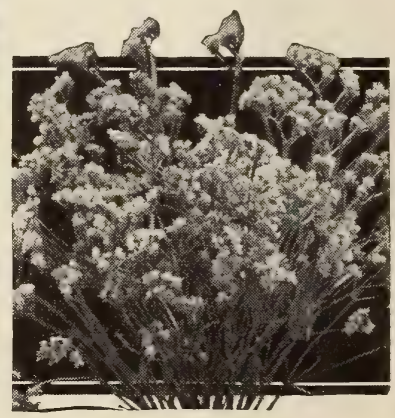

Statice (Sea Lavender) 


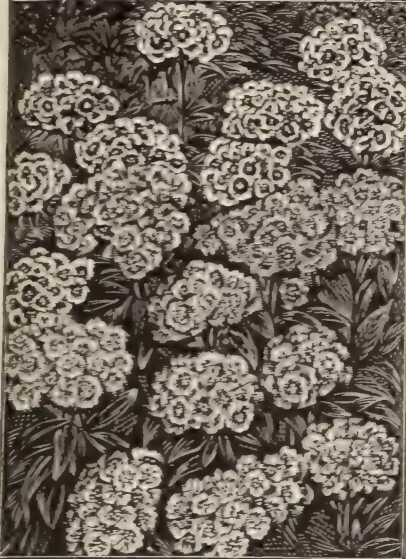

1928 Sweet William, Hardy Annual

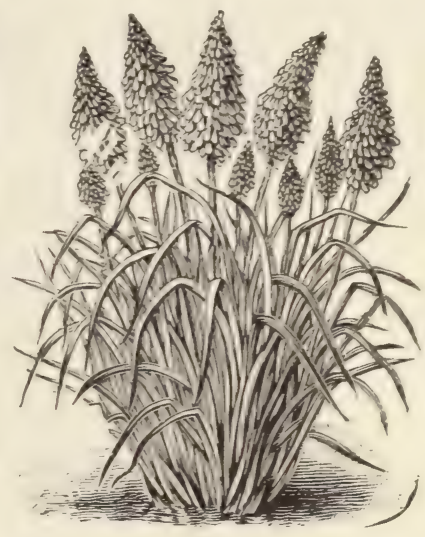

1946 Tritoma, Hybrida Express

\section{Sweet William}

Old-fashioned favorites.

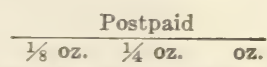

1936 Double Mixed, Beautiful colorings. $\$ 0.15 \quad \$ 0.25 \quad \$ 0.75$

1938 Single Mixed, All shades and mark-

1928 Hardy Annual, Finest Mixed Colors.

1940 Maule's Special Mixture......... $\quad .15 \quad .25 \quad .75$

Sweet Rocket (Hesperis matronalis)

Excellent for cutting.

1/4 oz. oz.

1941 Mixed Colors................. \$0.15 $\$ 0.30$

Thunbergia (Black-Eyed Susan)

A rapid growing vine.

1/4 oz. oz.

1942 Fine Mixed....................\$0.20 \$0.60

Tritoma (Red Hot Poker Plant)

Excellent for cut flowers.

1946 Hybrida, Express

$1 / 8$ oz. 1/4 oz. Oz.

$\$ 0.25 \quad \$ 0.40 \quad \$ 1.25$

\section{Umbrella Plant}

Showy pot plant.

1950 Cyperus alternifolius

\begin{tabular}{ccc}
\hline $1 / 8$ oz. & $1 / 4$ oz. & oz. \\
$\$ 0.20$ & $\$ 0.35$ & $\$ 1.00$
\end{tabular}

\section{Vinca}

Bushy plants for pots or beds.

1992 Mixed Colors, Free blooming..........\$0.25 $\quad \begin{gathered}\text { oz. } \\ \$ 0.70\end{gathered}$

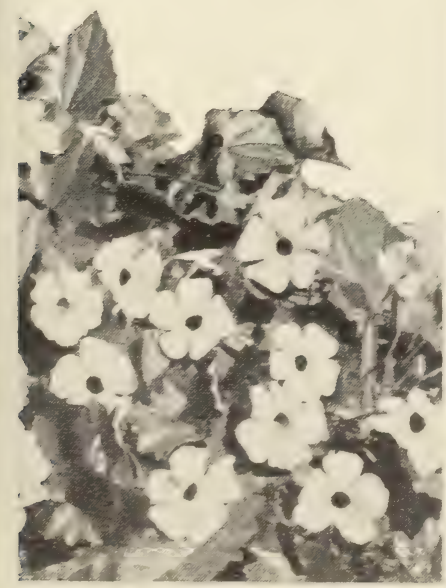

1942 Thunbergia, Fine Mixed

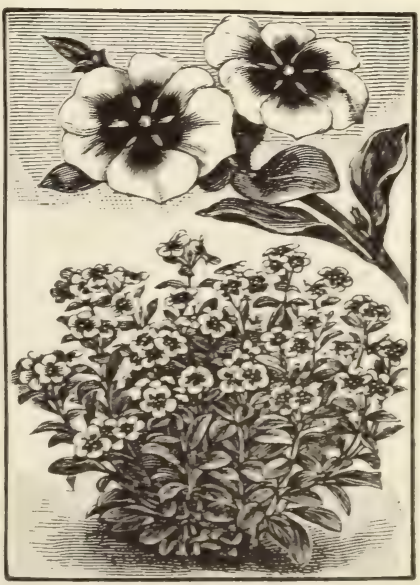

1992 Vinca, Mixed Colors

All flower seeds are sent postpaid at these special prices 


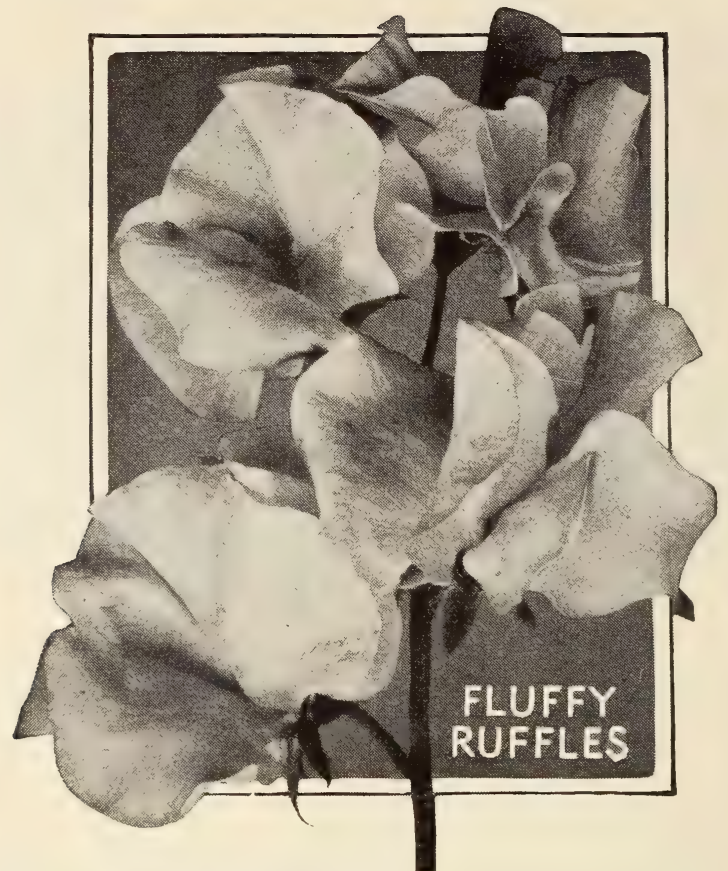

Giant Spencer Sweet Peas

The popular sort for outdoor summer flowering.

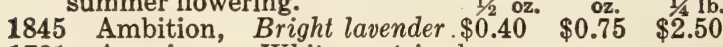

1781 America, White, striped crimson............... .15

1783 Ascot, Beautiful light pink. .15

1785 Avalanche, Pure white. .15

1823 Beatall, A glowing pink... .15

1825 Boy Blue, Bright blue..... . .15

1824 Bridesmaid, Cream-pink... .15

1849 Brilliant Rose, Bright rose 15

1858 Fire, Flaming scarlet-red... .20

1863 Floradale, Salmon-pink on cream............... .40

1846 Fluffy Ruffles, Rich rose pink .20

1862 Fordhook Orange, Brilliant

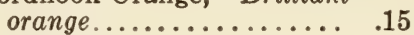

1787 Glen Eagles, Light blue.... .15

1789 Honour, Rich crimson..... .15

1791 Huntsman, Bright scarlet. 15

1793 Idyll, Cream-pink, shaded salmon...............

1805 Lady Ruffles, Soft pink on white. ... 1.25

1822 Lompoc, LaFrance pink shaded salmon.......... $40 \quad .45 \quad 2.50 \quad 7.50$

1882 Mammoth, Orange scarlet. .15

1886 Mary Pickford, A cream-pink .15

1887 Model Black seeded; white flowers................... .15

1795 Pinkie, Clear deep pink... .15

1904 Powerscourt, A pure lavender .15

1905 Royal Scot, A deep cerise. 15

1906 Ruffled Beauty, Lilac-pink. .30

1911 Ruffled Orchid, Rich lavender-pink............. .30

1900

Ruffled Rose, Rose with salmon sheen....... $\quad .30$

Sunkist, Cream, edged pink.

Tangerine Improved, Deep orange................ .15

Tom Webster, Lovely midblue............... 15

Giants Mixed........... . .10

$\begin{array}{lll}.10 & .30 \quad .85\end{array}$




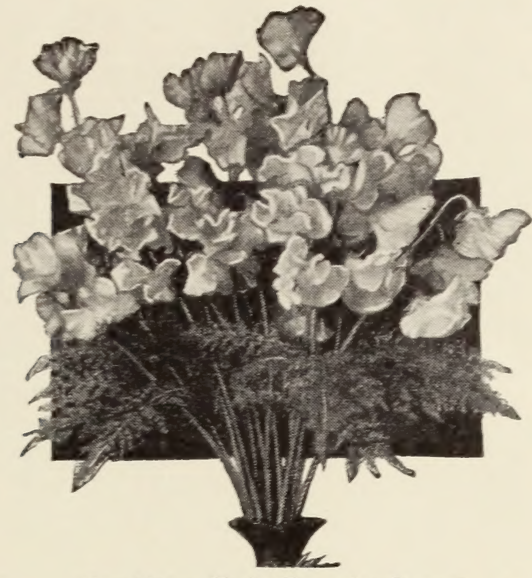

Sweet Peas, Early Flowering Spencer

\section{Sweet Peas, Early Flowering Spencer}

For forcing under glass for winter and early spring flowering.

\begin{tabular}{rrrr}
\multicolumn{4}{c}{ Postpaid } \\
\cline { 2 - 4 } $1 / 2$ oz. & oz. & $1 / 4$ lb. & \multicolumn{1}{c}{ lb. } \\
$\$ 0.25$ & $\$ 0.45$ & $\$ 1.50$ & $\$ 4.50$ \\
.30 & .50 & 1.65 & 5.00
\end{tabular}

1813 Bluebird, A violet-blue ....

orange..................... $.30 \quad .50 \quad 1.65 \quad 5.00$

1815 Gilda Gray, Salmon-cerise

1816 Early King, Crimson.....

1826 Chevalier, Beautiful rose blooms..................

1833 Mrs. Calvin Coolidge,
Salmon-pink..........
1834 Mrs. Kerr, The best salmon

1836 Lavender King, True deep lavender................

1837 Pink Profusion, Pink.

1838 Snowstorm I m p r o ve d, White................

1827 Illumination, Glowing

$.25 \quad .45 \quad 1.50 \quad 4.50$

$\begin{array}{llll}.30 & .50 & 1.65 & 5.00\end{array}$

1841 S u n burs t Improved, Cream and pink.........

$\begin{array}{llllll}1844 & \text { Early Flowering Mixed.... } & .15 & .25 & .75 & 2.25\end{array}$

\section{Verbena, Maule's Mammoth Show}

Flowers are of mammoth size. Unex-

celled for size, beauty, and richness of

color. Fine for pots, vases, or window

boxes.

1953 Rose-Pink............... )

1956 Lucifer

1957 Blue, White Eye...............

1958 Luminosa, Flame pink ............

$\begin{array}{lllll}1978 & \text { All Colors Mixed .................... } .25 & .40 & 1.25\end{array}$

1979 Royal Bouquet Mixed............ $\quad .50 \quad .85 \quad 2.50$

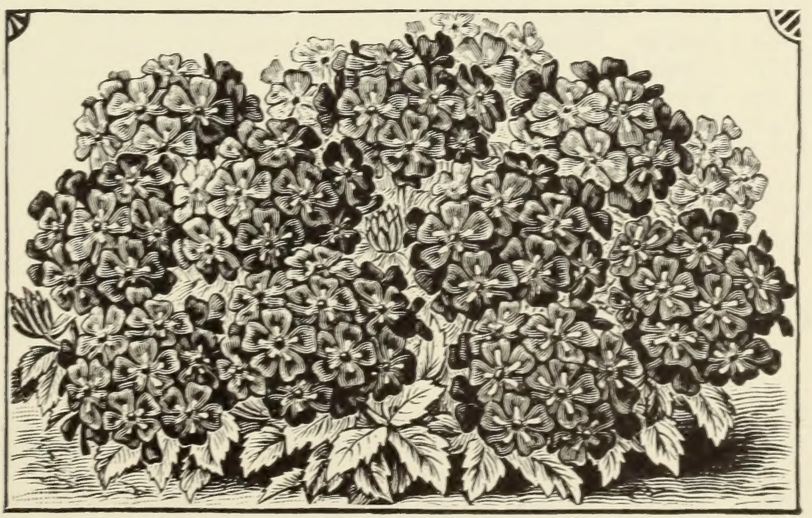

Verbena, Maule's Mammoth Show

All flower seeds are sent postpaid at these special prices 


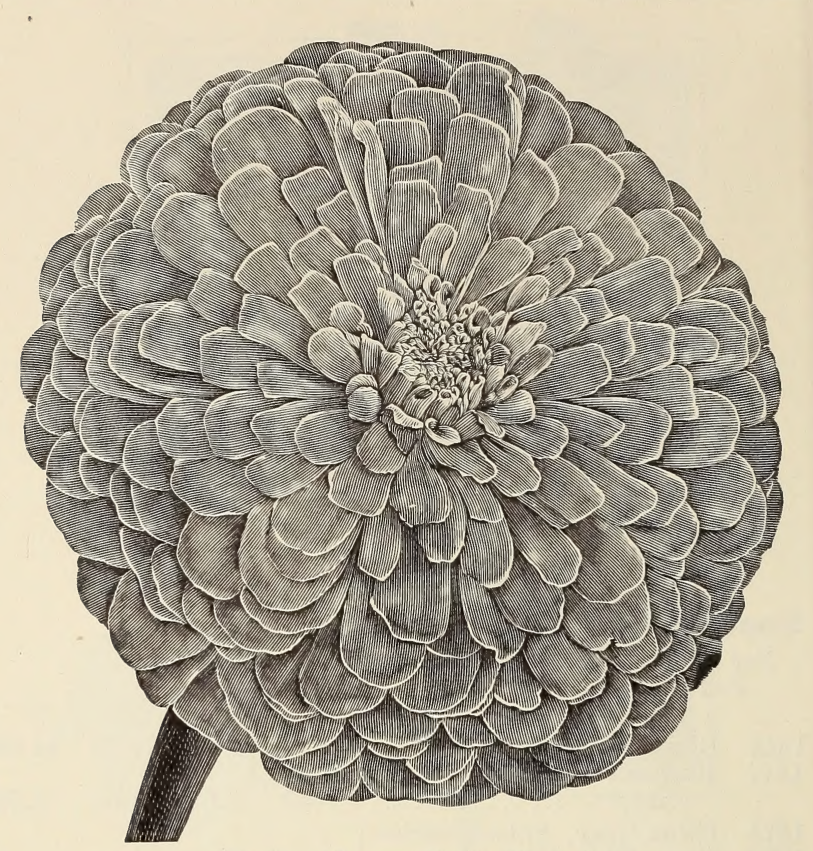

Zinnia. Mammoth Dahlia Flowered

\section{Zinnia, Mammoth Dahlia Flowered}

The largest and most attractive Zinnias.

2055 Crimson Monarch, Red..........

2056 Buttercup, Canary-yellow.........

2057 Illumination, Light rose..........

2058 Dream, Lavender................

2059 Golden State, Orange-yellow......

2060 Old Rose, Deep rose...........

2061 Oriole, Orange and gold............

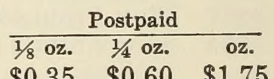

2062 Golden Dawn, Golden yellow......

$\begin{array}{lrr} & & \\ \$ 0.35 & \$ 0.60 & \$ 1.75\end{array}$

2064 Eldorado, Rose and salmon.......

2067 Luminosa, Pink, suffused salmon

2068 Old Gold, Deep orange...........

2065 All Colors Mixed, Dahlia Flowered. $\quad .20 \quad .35 \quad 1.00$

\section{Zinnia, California Giant Flowering Double}

Perfect form, round, full, and double. 2037 Brightness, Deep rose.......... ) 2038 Grenadier, Dark red.............. 2039 Lavender Gem, Light lavender.... 2041 Burnt Orange, Deep orange...... 2042 Crimson, Dark red............. 2043 Lemon Queen, Light yellow....... 2044 Purple, Deep purple............ 2045 Rose Queen, Bright rose.......... . 2046 Scarlet, Bright red ............. 2047 Flesh Pink, Light pink... . . . . . . 2048 White, Pure white.............. 2049 Deep Salmon-Rose............. 2050 Golden Yellow, Deep yellow...... 2052 Sulphur Yellow, Pale yellow..... 2053 Orange King, Bright orange....... 2054 Mixed Colors, Giant Flowering. . .

\section{Zinnias, Miscellaneous}

All double flowering. Excellent for cutting or for bedding.

2036 Double Curled and Crested, Mixed. 2040 Dwarf Double, All Colors, Mixed. . 2066 Double Quilled, Mixed Colors...... \$0.2 2070 Double Picotee, Mixed Colors...... 2076 Double Lilliput, Mixed Colors......

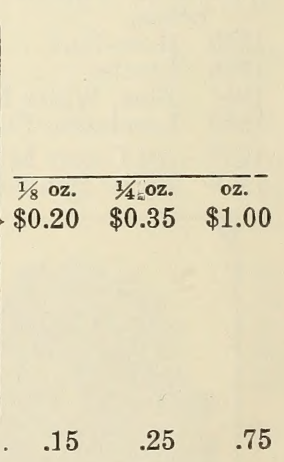

$\$ 0.20 \quad \$ 0.35 \quad \$ 1.00$ 


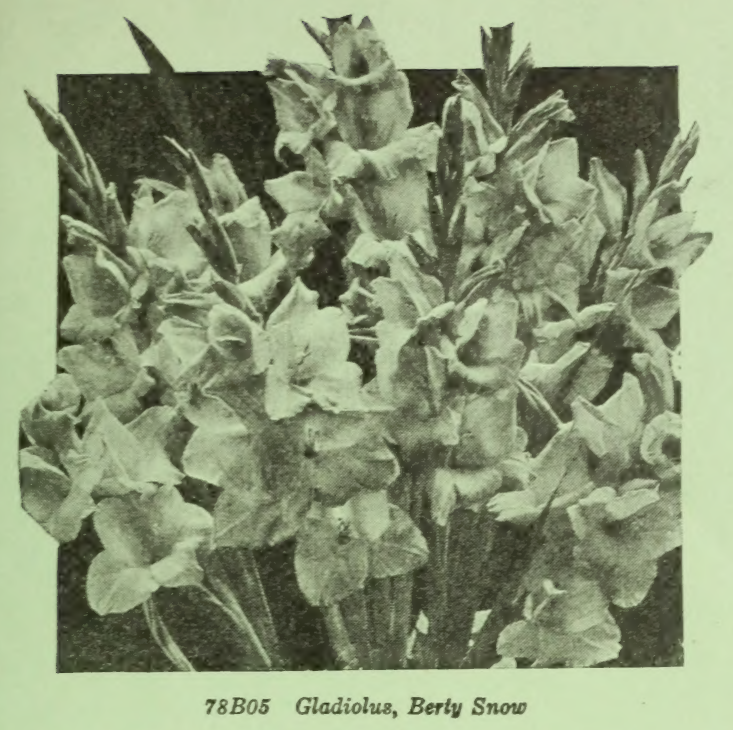

\section{Maule's Exhibition Gladiolus First Size Bulbs}

78B01 America, Soft shell pink......... \$0.25

78B00 Alice Tiplady, Bright orange-yellow .25

78B05 Berty Snow, Light rosy lavender... $\quad .70$

78B07 Baron Hulot, Violet-purple....... .60

78B13 Cowee's Scarlet Wonder, Scarlet. . .35

78B16 Crimson Glow, Brilliant crimson. .35

78B50 Dr. F. E. Bennett, Peach-red overlaid scarlet.................

$78 \mathrm{~B} 53$

$78 \mathrm{~B} 54$

$78 \mathrm{~B} 31$

$78 \mathrm{~B} 37$

$78 \mathrm{~B} 43$

$78 \mathrm{~B} 44$

$78 \mathrm{~B} 56$

Giant Nymph, Light rose-pink. .

Golden Frills, Daffodil yellow........

Golden Measure, Golden yellow... . .35

Halley, Salmon-pink ............. .25

Le Marechal Foch, Flesh-pink.... .25

Los Angeles, Orange, tinted pink.:
Mrs. Leon Douglas, Rose, striped orange-scarlet..............

Mrs. F. C. Peters, Soft rosy lavender

$78 \mathrm{~B} 64$

Mrs. Von Konynenburg, Light blue

Peace, Pure white...............

$78 \mathrm{~B} 73$

$78 \mathrm{~B} 76$

$78 \mathrm{~B} 60$

$78 \mathrm{~B} 61$

$78 \mathrm{~B} 86$

$78 \mathrm{~B} 87$

$78 \mathrm{~B} 89$ Purple Glory, Purple-maroon..... . . .35 Rose Ash, Old rose............ . .35 Souvenir, Rich golden yellow...... .25 The Orchid, Pale lavender...... $\quad .70$ W. H. Phipps, Pink, striped salmon. .45 Maule's Extra Fine Mixed Primulinus Varieties, Mixed ..... . .30

Not Prepaid

$\$ 1.75 \$ 15.00$

$1.75 \quad 15.00$

$5.00 \quad 45.00$

$4.00 \quad 35.00$

$2.75 \quad 25.00$

$2.50 \quad 20.00$

$3.00 \quad 25.00$

$2.50 \quad 20.00$

$2.75 \quad 25.00$

2.7525 .00

$\begin{array}{ll}1.75 & 15.00\end{array}$

$1.75 \quad 15.00$

$2.50 \quad 20.00$

$2.50 \quad 20.00$

2.7525 .00

$3.50 \quad 30.00$

$2.50 \quad 20.00$

$2.75 \quad 25.00$

$2.50 \quad 20.00$

1.7515 .00

$5.00 \quad 45.00$

$3.00 \quad 25.00$

$2.50 \quad 20.00$

$2.00 \quad 15.00$

\section{MISCELLANEOUS BULBS}

\section{Amaryllis}

70B02 Equestris, Orange scarlet.

$\frac{\text { Not Prepaid }}{3 \quad \text { Doz. } 100}$

70B04 Giant American Hybrid, Mixed.. $1.25 \quad 4.50 \quad 35.00$

70B05 Johnsoni, Velvety crimson......... $1.00 \quad 3.50 \quad 25.00$

\section{Begonias Tuberous-rooted}

70B43 Single, Mixed, All shades......... . .70 $\quad 2.50 \quad 18.00$ 70B44 Double, Mixed, All colors......... $\quad .70 \quad 2.50 \quad 18.00$

70B38 Caladium, Fancy leaved........... $\quad .85 \quad 3.00 \quad 22.00$ 70B40 White Calla Lily, Large white.... $\quad .85 \quad 3.00 \quad 22.00$ $70 \mathrm{~B} 42$ Yellow Calla Lily, Golden yellow. . $1.00 \quad 3.50 \quad 26.00$

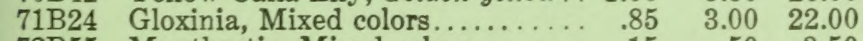
$72 \mathrm{~B} 55$ Montbretia, Mixed colors......... $\quad .15 \quad .50 \quad 3.50$

$$
\frac{\text { Not Prepaid }}{\text { Doz. } 100 \quad 1000}
$$

74B18 Tuberose, Double, white, long stems $\$ 0.50 \$ 03.00 \$ 25.00$

NOTE-You may order 6 Bulbs or Roots of a kind at dozen rate; 25 or over of a kind at 100 rate, 200 or over of a kind at 1,000 rate. 


\section{Maule's Seeds}

Once Grown-Always Grown
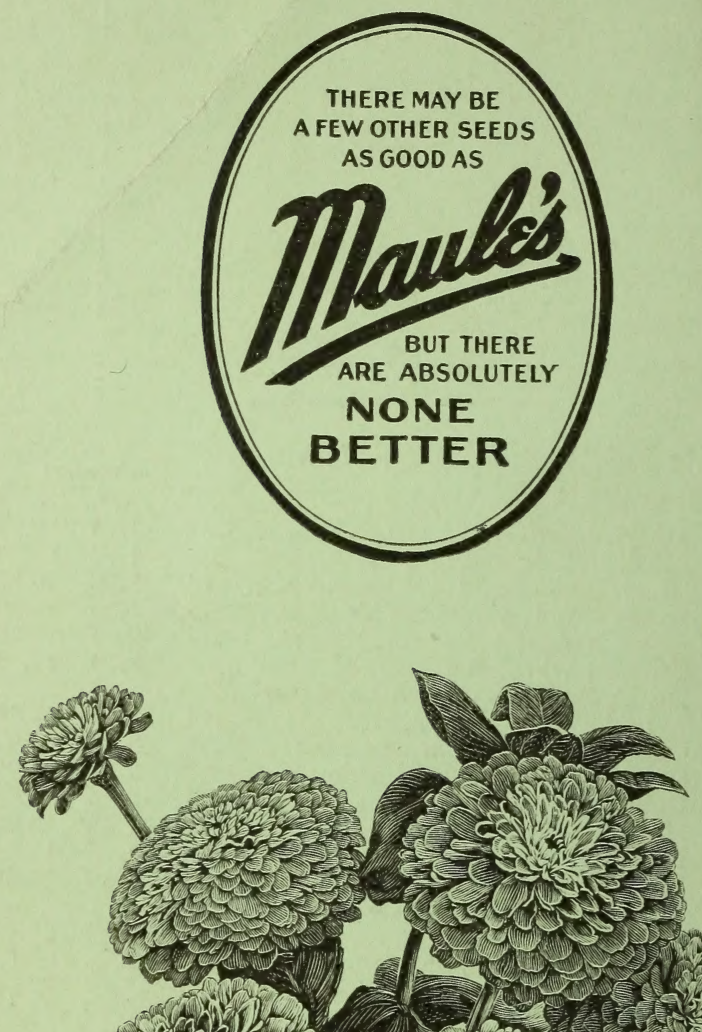

N(a) 5 (1)

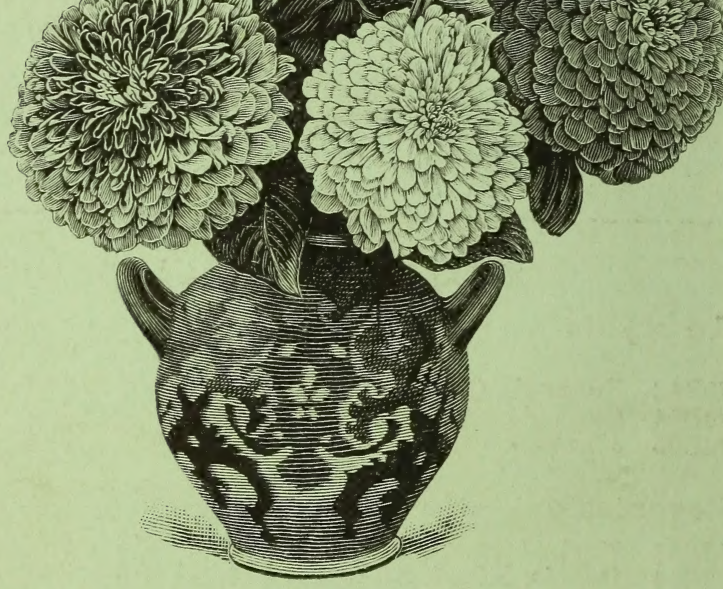

Dahlia Flowered Zinnias

\section{WM. HENRY MAULE}

"The Old Reliable Seed House"

1220 Spring Garden Street 